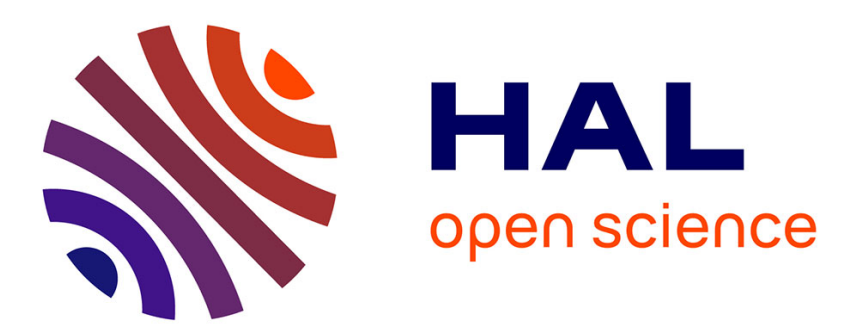

\title{
A novel Ka-band chirped-pulse spectrometer used in the determination of pressure broadening coefficients of astrochemical molecules
}

Thomas Hearne, Omar Abdelkader Khedaoui, Brian Hays, Théo Guillaume, Ian R Sims

\section{To cite this version:}

Thomas Hearne, Omar Abdelkader Khedaoui, Brian Hays, Théo Guillaume, Ian R Sims. A novel Ka-band chirped-pulse spectrometer used in the determination of pressure broadening coefficients of astrochemical molecules. Journal of Chemical Physics, 2020, 153 (8), pp.084201. 10.1063/5.0017978 . hal-02922763

\section{HAL Id: hal-02922763 \\ https://hal-univ-rennes1.archives-ouvertes.fr/hal-02922763}

Submitted on 26 Aug 2020

HAL is a multi-disciplinary open access archive for the deposit and dissemination of scientific research documents, whether they are published or not. The documents may come from teaching and research institutions in France or abroad, or from public or private research centers.
L'archive ouverte pluridisciplinaire HAL, est destinée au dépôt et à la diffusion de documents scientifiques de niveau recherche, publiés ou non, émanant des établissements d'enseignement et de recherche français ou étrangers, des laboratoires publics ou privés. 


\title{
A novel Ka-band chirped pulse spectrometer used in the determination of pressure broadening coefficients of astrochemical molecules
}

\author{
Authors: \\ Thomas S. Hearne, Omar Abdelkader Khedaoui, Brian M. Hays, Théo Guillaume, and lan R. Sims* \\ electronic mail: ian.r.sims@univ-rennes1.fr
}

Affiliation:

Univ Rennes, CNRS, IPR (Institut de Physique de Rennes) - UMR 6251, F-35000 Rennes, France

\begin{abstract}
:
A novel chirped-pulse Fourier transform microwave (CP-FTMW) spectrometer has been constructed to cover the Ka-band ( $26.5 \mathrm{GHz}-40 \mathrm{GHz}$ ) for use in the CRESUCHIRP project, which aims to study the branching ratios of reactions at low temperatures using the chirped-pulse in uniform flow (CPUF) technique. The design takes advantage of recent developments in radio-frequency components; notably high-frequency, highpower solid-state amplifiers. The spectrometer had a flatness of $5.5 \mathrm{~dB}$ across the spectral range, produced harmonic signals below $-20 \mathrm{dBc}$, and the recorded signal scaled well to 6 million averages. The new spectrometer was used to determine pressure broadening coefficients with a helium collider at room temperature for three molecules relevant to astrochemistry, applying the Voigt function to fit the magnitude of the Fourier-transformed data in the frequency domain. The pressure broadening coefficient for OCS was determined to be $(2.45 \pm 0.02) \mathrm{MHz} \mathrm{mbar}^{-1}$ at room temperature, which agreed well with previous measurements. Pressure broadening coefficients were also determined for multiple transitions of vinyl cyanide and benzonitrile. Additionally, the spectrometer was coupled with a cold, uniform flow from a Laval nozzle. The spectrum of vinyl cyanide was recorded in the flow, and its rotational temperature was determined to be $(24 \pm 11) \mathrm{K}$. This temperature agreed with a prediction of the composite temperature of the system through simulations of the experimental environment coupled with calculations of the solution to the optical Bloch equations. These results pave the way for future quantitative studies in low-temperature and high-pressure environments using CP-FTMW spectroscopy.
\end{abstract}




\section{INTRODUCTION}

The interstellar medium, the material that forms stars and planets and exists between them, is chemically rich with over 200 molecules so far identified in different areas of space. The interactions between these molecules occur at very low temperatures and pressures, over long timescales, and occasionally with the influence of high-energy radiation. To accurately model these environments, laboratory data are needed for these interactions at similar conditions to those found in space. Amongst such data are the branching ratios for chemical reactions that have more than one product channel. Few experiments exist that are capable of determining branching ratios, especially at the low temperatures of the interstellar medium. ${ }^{1}$ The aim of the CRESUCHIRP project is to determine these ratios by coupling the well-established CRESU (Cinétique de Réaction en Ecoulement Supersonique Uniforme, or reaction kinetics in uniform supersonic flow) technique ${ }^{1,2}$ for studies of cold chemical kinetics with chirped-pulse Fourier-transform microwave (CPFTMW) spectroscopy. ${ }^{3}$ The combination of these techniques has been designated as chirped pulse in uniform flow (CPUF). ${ }^{4,5}$ To this end, a novel CP-FTMW spectrometer operating in the Ka-band (26.5 - 40.0 $\mathrm{GHz}$ ) has been designed, built and tested. The Ka-band spectrometer complements an existing E-band spectrometer, which was recently built for the CRESUCHIRP project. ${ }^{6}$

Rotational spectroscopy is a powerful tool for product identification: each molecule has a unique rotational spectrum which is specific to a particular molecular isomer, conformer, isotopologue and vibrational mode. Furthermore, the CP-FTMW method can cover a wide spectral range with a single scan and can rapidly target specific transitions. ${ }^{3}$ These traits, combined with the well-established response of molecular dipoles to external stimulation, ${ }^{7}$ make CP-FTMW spectroscopy an attractive tool for detecting diverse species simultaneously in a quantitative manner. This has been demonstrated in earlier work, where the CPUF technique was used to measure the product branching ratios of the reaction between $\mathrm{CN}$ and propyne in a uniform CRESU flow, ${ }^{8}$ and, in a quasi-uniform flow, the photodissociation of isoxazole ${ }^{9}$ and the photodissociation of the propargyl radical ${ }^{10}$. However, there are a few challenges associated with CP-FTMW spectroscopy. Firstly, a single CP-FTMW scan is not very sensitive, so spectra must be compiled from averages of up to millions of scans. Therefore, maintaining phase-stability between the various components involved is of utmost importance. Secondly, the power and duration of the excitation pulse needs to be finely tuned to elicit the best response from the molecular dipoles. ${ }^{11}$ Thankfully, recent advances in microwave technology have greatly benefitted the field of CP-FTMW spectroscopy. For example: faster switches, lower-noise/higher-gain low-noise amplifiers (LNAs), faster data acquisition devices, and more powerful, faster-switching broadband power amplifiers have all made CP-FTMW spectroscopy a competitive tool for quantitative analysis. 
In order for CP-FTMW spectroscopy to be effective for quantitative studies, it is essential to know some spectroscopic information about the system to be studied. Primarily, the transitions to be probed need to be well documented, with data on their frequencies, quantum numbers and intensities. Additionally, for experiments that maintain a thermodynamic equilibrium through collisions with a buffer gas, such as those to be performed in CRESU flows, data on the collisional broadening of the rotational transitions is vital for determining the feasibility of a study. The shape of the free induction decay (FID) can be described by the well-known Voigt profile; this includes two decay parameters which describe the half-width at halfmaximum (HWHM) of the Gaussian component of the decay; $\Delta v_{D o p p}$, and the Lorentzian component of the decay; $\Delta v_{\text {pres. }}$. The $\Delta v_{\text {Dopp }}$ component is an inhomogenous parameter directly related to Doppler broadening. On the other hand, $\Delta v_{\text {pres }}$ is a homogenous parameter directly related to collisional or pressure broadening. In CP-FTMW experiments, collisions degrade the polarization of the molecular sample induced by the chirped pulse. Collisional broadening affects the spectra of all molecules in high-pressure environments, and as such these parameters are important in the fields of atmospheric chemistry, and exoplanet chemistry, as well as in astrochemistry.

Collisional broadening data are not readily available for many species of astrochemical interest, as these types of experiments at relatively high pressures and low temperatures are a recent development in the application of CP-FTMW spectroscopy. Carbonyl sulfide (OCS), besides its relevance as a species detected in interstellar space, ${ }^{12}$ and planetary atmospheres, ${ }^{13}$ is a useful molecule for benchmarking CP-FTMW spectrometers, and has a well-defined transition $(J=3-2)$ within the Ka-band. This transition has been studied in the context of pressure broadening with a helium buffer gas by Story et al. who observed pressure broadening in a microwave absorption experiment. ${ }^{14}$ Numerous other transitions of OCS have also had their broadening coefficients with helium determined. ${ }^{15,16,17,6}$ Vinyl cyanide (acrylonitrile, $\left.\mathrm{CH}_{2} \mathrm{CHCN}\right)^{18}$ and benzonitrile $\left(c-\mathrm{C}_{6} \mathrm{H}_{5} \mathrm{CN}\right)^{19}$ are both molecules that have been detected in interstellar space, but little is known about their pressure broadening behavior. Cyanide-containing molecules are ideal for rotational spectroscopy as they often have a strong dipole moment, examples that have had their collisions with helium studied include: methyl cyanide $\left(\mathrm{CH}_{3} \mathrm{CN}\right),{ }^{20,21}$ cyanoacetylene $\left(\mathrm{HC}_{3} \mathrm{~N}\right),{ }^{22-25}$ and hydrogen cyanide $(\mathrm{HCN})^{26-28}$. With the goal in mind of providing data useful to CPUF experiments, the important transitions within the Ka-band for the species OCS, vinyl cyanide, and benzonitrile have been identified and characterized in order to determine their pressure broadening coefficients. Vinyl cyanide was also studied in a cold, high-pressure CRESU flow, with its rotational temperature determined through CP-FTMW spectroscopy. 


\section{EXPERIMENTAL}

\section{A. Ka-band spectrometer}

In designing the spectrometer, priority was given to phase-stability and the signal-to-noise ratio (SNR), utilizing the latest technology available. The design drew inspiration from the Ka-band spectrometer detailed by Zaleski et $a l,{ }^{29}$ and the lower frequency $(8.7-18.3 \mathrm{GHz})$ spectrometer detailed by Reinhold et al. ${ }^{30}$ Two different configurations of the spectrometer have been tested: one with a wide-band oscilloscope for data capture and a second featuring a fast digitizer card for data capture, which provides a speed improvement at the cost of bandwidth. The schematics of the two configurations are shown in figure 1.

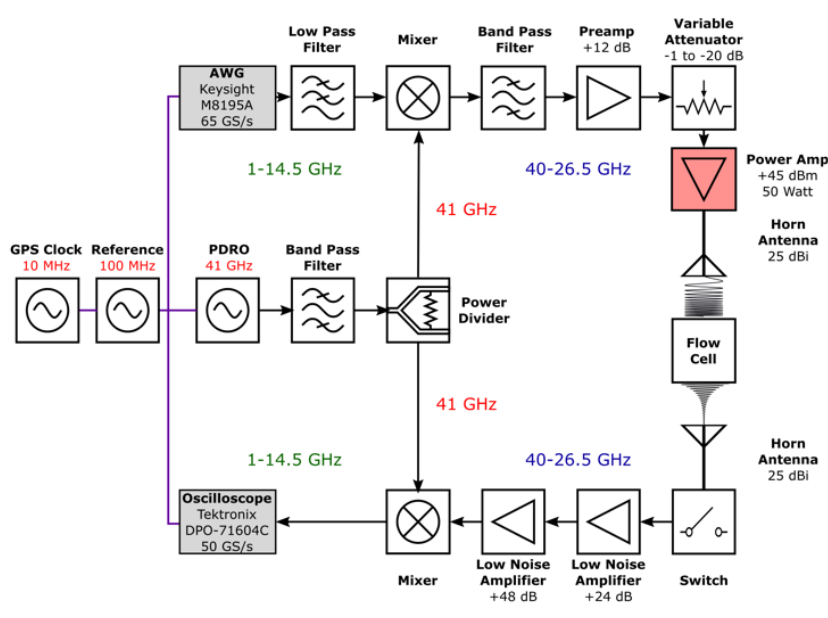

(a) - Configuration 1

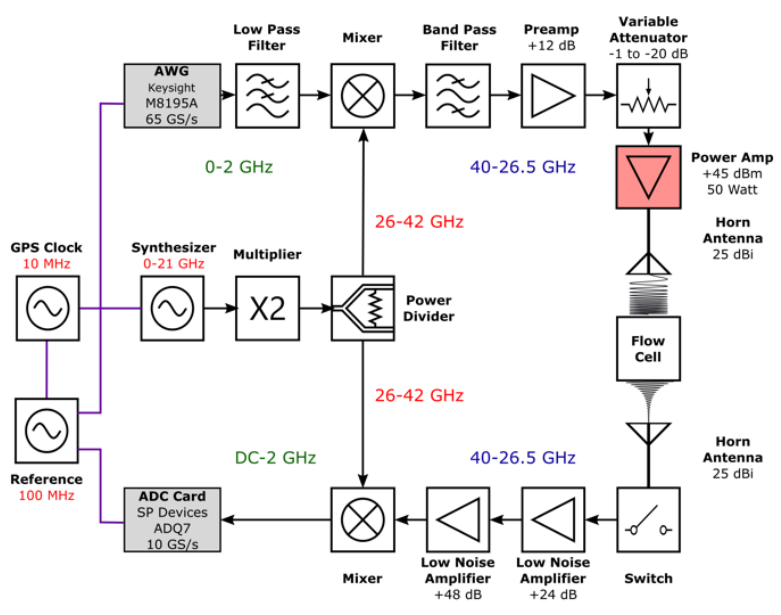

(b) - Configuration 2

Figure 1. Part (a) shows the testing configuration of the spectrometer using an oscilloscope as the data capture device. On the right in part (b) is the configuration used in the pressure broadening experiments with the digitizer in use as the data capture device.

The first configuration (configuration 1), which is primarily used for testing purposes, begins with chirped pulses generated by a Keysight arbitrary waveform generator (AWG - M8195A) from 1-16 GHz. The chirped pulses are up-converted to the Ka band through a Marki double-balanced mixer (MM1-1850H), using a phase-locked dielectric resonant oscillator (PDRO - Microwave Dynamics PLO-2000) providing a $41.1 \mathrm{GHz}$ single frequency as the local oscillator (LO) which is filtered by a bandpass filter (Reactel - 4W8-41G-×200 K11) with a nominal bandwidth of $400 \mathrm{MHz}$. The upconverted pulse passes through an RF-Lambda preamplifer (R24G40GSC) and a variable attenuator (RLC AV-26540) for careful level matching to the power amplifier. The power amplifier is a $50 \mathrm{~W}$ solid state amplifier manufactured by Meuro (MPH260400G4547R), which represents the cutting-edge in high-frequency, high-power solid state amplification. To the authors' knowledge, this study presents the first example of a high-power, Ka-band solid state amplifier used for 
molecular spectroscopy. The amplifier is fast switching (around $30 \mathrm{ns)}$ ) and able to operate at a specified powered output duty cycle of up to $30 \%$. The output of the amplifier is coupled to a $25 \mathrm{dBi}$ gain horn antenna (Sage SAR-2507-28-S2), which directs radiation through a Teflon window into a $1.5 \mathrm{~m}$ long by 0.15 $m$ diameter vacuum chamber, containing the species of interest. ${ }^{6}$ The interior of the vacuum chamber is lined with microwave-absorbent material (Laird Eccosorb HR-10) in order to dampen reflections of the excitation pulse within the cell.

At the opposite end of the flow chamber, radiation exits through another Teflon window, before it is received by a second $25 \mathrm{dBi}$ rectangular horn. The signal then passes through a waveguide variable attenuator (Sage STA-30-28-M2) which is used to protect the receiver circuit when testing the switch timings. The switch itself (Millitech PSP-28-SIAHF) is immediately behind the attenuator; it has a rise time (to $95 \%$ transmission) of $70 \mathrm{~ns}$, a fall time of around $20 \mathrm{~ns}$, and isolates from $40-50 \mathrm{~dB}$ across the Ka-band. The switch is precisely timed to absorb signal when the high-power excitation pulse is active, and to pass signal immediately after the end of the pulse, to allow the FID to proceed to the LNA stage. After the switch, the signal is amplified by two cascaded, coaxial Ka-band LNAs (RF-Lambda R24G40GSB, Miteq LNA-40-2600400035-15P) with a combined gain of $64 \mathrm{~dB}$. The amplified signal is downconverted through an identical Marki mixer to the upconversion stage, using the same PDRO LO source. By using the same LO source on the upconversion and the downconversion stage, phase-noise introduced by the LO effectively cancels itself out. The intermediate frequency (IF) from the mixer is finally passed directly into the oscilloscope for measurement.

The master clock for the spectrometer is provided by a GPS-disciplined rubidium clock (SRS FS740) which outputs a $10 \mathrm{MHz}$ timing signal. This signal is converted to a $100 \mathrm{MHz}$ timing signal by an ovencontrolled crystal oscillator (OCXO) coupled with a distributing amplifier from Precision Test Systems (Precision Test Systems GPS10-eR-50). The $100 \mathrm{MHz}$ signal will provide better stability when scaled up to the clock frequencies of the AWG, oscilloscope, and PDRO, which are all locked to this signal. The AWG has 2 marker channels available which are used to trigger the spectrometer. The first channel is sent directly to the recording device (oscilloscope or ADC card) to trigger the acquisition. The second channel is fed to a digital delay generator (DDG - BNC 575), timed by the $10 \mathrm{MHz}$ clock signal, which triggers the non-phasecritical parts of the experiment, namely the power amplifier and the receiver-side switch.

A second configuration of the spectrometer (configuration 2), which was used to record all the spectral data presented here, employs a fast digitizer card for data acquisition synchronized to the $100 \mathrm{MHz}$ signal. This card from SP-Devices (ADQ7DC-PCIE-FWATD) operates at $10 \mathrm{GS} / \mathrm{s}$, using dual interleaved ADC chips, with a nominal bandwidth of $2.5 \mathrm{GHz}$. The card can record a frame of up to $200 \mu \mathrm{s}$, with a fast repetition rate of up to $30 \mathrm{kHz}$. The onboard field-programmable gate array (FPGA) can sum up to 262,144 
repetitions, at which point the data is transferred to the host computer and the card re-arms itself. This allows for a substantial improvement in recording speed over the oscilloscope, which can record typically at a rate of 30 acquisitions per second in these conditions. However, as the ADC card can only record up to 2.5 $\mathrm{GHz}$ of bandwidth at the $3 \mathrm{~dB}$ level, it is necessary to set the LO frequency in the downconversion stage to within $2.5 \mathrm{GHz}$ of the transition to be probed in order for the FID to be detectable by the card. For this purpose, the output from a $0.5-21 \mathrm{GHz}$ Signal Core synthesizer (SC5511A) was used, after doubling in a Marki frequency doubler (ADA-1020), to provide an LO tunable within the range of $26-41 \mathrm{GHz}$. For the phasebenefit of using the same LO source on both sides of the frequency conversion, the doubled synthesizer is used for both up- and down-conversion. The use of a movable LO results in the acquisition of dual-sideband spectra. Care was taken to ensure that different transitions do not overlap in the acquired spectra. A second drawback of the $A D C$ card is that it introduces a strong spurious signal at $2.5 \mathrm{GHz}$ due to the interleaving of its dual ADC processors. This spur, and other electrically-induced spurs, are efficiently removed from the resulting spectrum by using a correlation filter, built from the final part of the acquisition, well after any FIDs have decayed away.

\section{B. Gas-phase studies}

For the determination of pressure broadening parameters, mixtures of the species of interest and helium (Air Liquide - 99.995\% purity) were made up in a large mixing vessel, before being introduced into the flow cell via a flow controller (Brooks GF080CXXC). Mixtures of 13 mbar of OCS (Sigma Aldrich - 97.5\% purity) with 1.41 bar of He (0.92 \% by pressure), 14 mbar of vinyl cyanide (Sigma Aldrich - 99\% purity) with 1.48 bar of $\mathrm{He}$ ( $0.95 \%$ by pressure) and 1.3 mbar of benzonitrile (Acros Organics $-99 \%$ purity) with 0.15 bar of $\mathrm{He}(0.87 \%$ by pressure) were used for these experiments. The liquid species, vinyl cyanide and benzonitrile, were subjected to multiple freeze-pump-thaw cycles before each use. Additional helium was flowed into the cell via another flow controller (Brooks GF080CXXC) to vary the pressure within the cell. For self-broadening measurements, benzonitrile vapor was introduced into the cell via a dosing valve which allowed fine control of the total cell pressure of benzonitrile. The cell was pumped by a turbomolecular pump, backed by a scroll pump, and the pressure determined by a capacitance manometer (Brooks XacTorr $\mathrm{CMXO)}$. All flow cell experiments were performed at room temperature, which was regulated at $21^{\circ} \mathrm{C}$.

For the CRESU experiments a flow of liquid vinyl cyanide set by a Coriolis flow meter (Bronkhorst mini CORI-FLOW ML120 V00) to $5 \mathrm{~g} \mathrm{~h}^{-1}$ was vaporized through a controlled evaporative mixer (CEM Bronkhorst W-202A-222-K) with the vapor transported by a flow of 5 SLM (standard liters per minute) of a helium carrier gas supplied by a separate mass flow controller (Bronkhorst EL-FLOW Prestige). ${ }^{31}$ The evaporated mixture was injected along with an additional supply of helium buffer gas, which was continuously adjusted to maintain a constant pressure of $97.73 \mathrm{mbar}$, as measured by a capacitance 
manometer (Brooks XacTorr CMX0), within a reservoir mounted in the experimental chamber. The gas in the reservoir continuously expanded into the chamber through a Laval nozzle. The experimental chamber was maintained at a pressure of 0.113 mbar by a series of roots pumps. The nozzle was characterized by 2-D impact pressure measurements using a pitot tube, displayed in Figure 2, showing a uniform flow core with an average temperature of $(18.7 \pm 0.5) \mathrm{K}$ and pressure of $(0.113 \pm 0.002)$ mbar. Computational fluid dynamics (CFD) calculations were performed using the rhoCentralFoam solver ${ }^{32}$ in the openFOAM package $\mathrm{e}^{33}$ to further characterize the nozzle flow and in particular the properties of the boundary layers. A comparison to the Pitot tube measurements is shown in Figure 2. The periodic variation in the parameters of the flow is due to small, stationary expansion/compression waves due to a difference between the core pressure and the ambient pressure in the chamber. Two $17 \mathrm{dBi}$ Ka-band horns were mounted in the chamber around 20 $\mathrm{cm}$ apart, defining a transverse axis that intersected the center of the uniform flow at $18 \mathrm{~cm}$ from the nozzle exit. A Kapton waveguide vacuum window was placed behind each horn, and the interior of the chamber was well-covered with microwave absorbent foam (Laird Eccosorb HR-10). The CRESU flow was probed with the Ka-band spectrometer using 10 ns single-frequency pulses targeting particular transitions of vinyl cyanide, with each FID averaged 500,000 times. The peak response of the strong vinyl cyanide transitions was for a pulse length of around 15 ns. A shorter pulse length benefitted from fewer reflections from the excitation pulse being present in the recorded data. A blank performed without vinyl cyanide flow but otherwise under identical conditions was subtracted from the recorded signals. 
(a) - Simulated Density

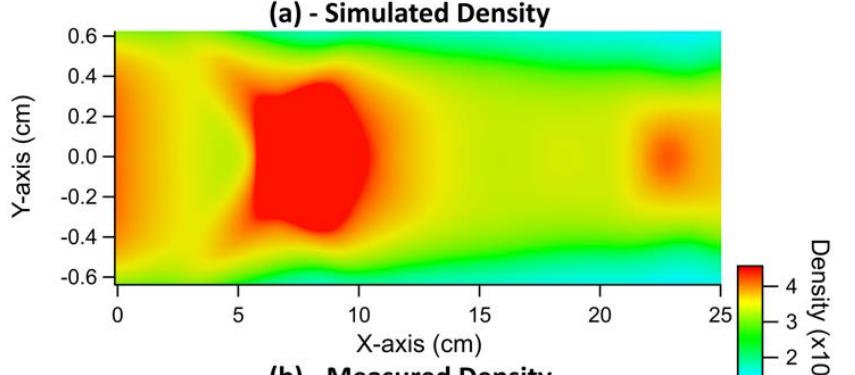

(b) - Measured Density
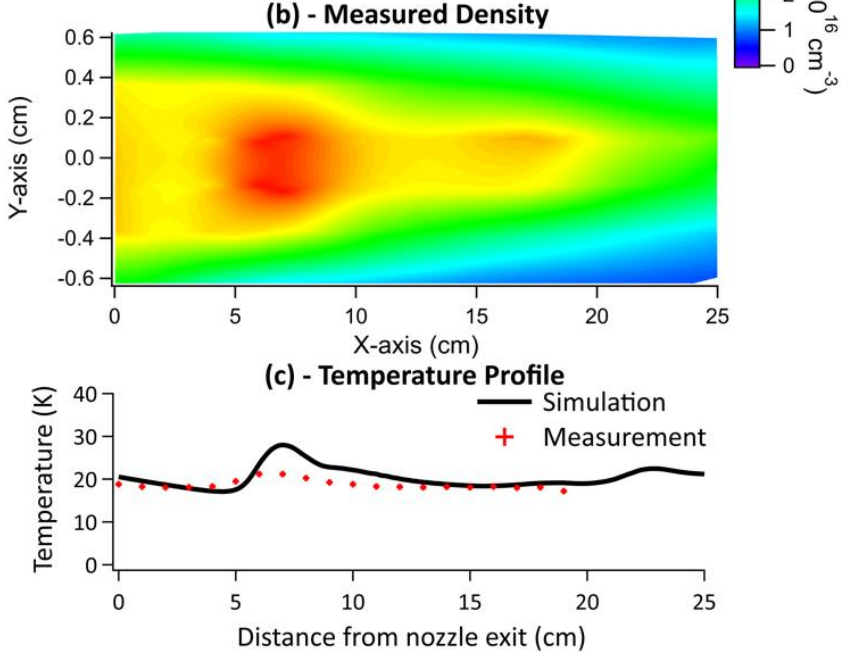

Figure 2. Characterization of the flow through the Laval nozzle used in the CRESU experiments. Part (a) shows the flow density as determined by a CFD simulation using the rhoCentralFoam solver included in the openFOAM software. Part (b) shows the flow density as determined by Pitot tube measurements of impact pressure at various distances. Part (c) shows the temperature profile of the flow along its axis from the exit of the nozzle, comparing the simulated and the Pitot tube results.

The recorded FIDs were analyzed in the manner previously detailed for a time-domain Voigt profile. ${ }^{6}$ However, transitions occurring in crowded spectral regions were difficult to fit in the time domain. In these cases, recorded FIDs were converted to frequency-domain spectra by taking the absolute value of a fast Fourier transform (FFT). The FFT was performed with a square window gated over the FID, avoiding the parts of the FID interfering with reflected light resulting from the excitation pulse. The magnitude of the FFT was used as it is considered to be complicated to correct for the phase of the individual time-domain signal components in CP-FTMW spectroscopy, which can be done to produce absorption-only spectra. ${ }^{34,35}$ In the case where the magnitude of the FFT is used to produce frequency-domain spectra, both the absorption line profile and the dispersion line profile must be considered. The frequency-domain lines from these experiments were fit to the equation of the magnitude of the complex Voigt function, which includes the absolute value of the real component of the Faddeeva function with the imaginary component of the Faddeeva function: 


$$
f\left(x, a, \Delta v_{\text {pres }}, \delta, \sigma\right)=a_{1} \frac{\left|w\left(\frac{x-\delta+\Delta v_{\text {pres }} i}{\sigma \sqrt{2}}\right)\right|}{\sigma \sqrt{2 \pi}}
$$

Where $x$ is the frequency, $\sigma=\Delta v_{D o p p} / V(2 \ln (2)), w()$ is the Faddeeva function, $\delta$ is a frequency offset, $a_{1}$ is a scaling constant corresponding to the integrated intensity, and $\Delta v_{\text {pres }}$ and $\Delta v_{\text {Dopp }}$ are the half width at half max (HWHM) values of the Lorentzian decay component of the FID and the Gaussian decay component of the FID respectively. This form of the Voigt function includes both the absorption component and the dispersion component of the complex line-shape, the real value giving the absorption Voigt profile, and the imaginary value giving the dispersion contribution.

The fitting for both the time-domain and the frequency-domain data was tested using the Levenberg-Marquardt method, with the global minimum verified using the AMPGO (adaptive memory programming for constrained global optimization) method, ${ }^{36}$ implemented in the Lmfit Python package. ${ }^{37}$ Transitions which consisted of several unresolved hyperfine components were fit to a summation of multiple Voigt profiles. The variation in transition frequency for different hyperfine components is considered to be negligible considering the scale of the change compared to the size of Doppler broadening in these experiments, therefore Doppler broadening was constrained to a single value for each hyperfine transition. Furthermore, the pressure broadening parameters of the different hyperfine components were assumed to be equal, for this analysis. The total uncertainty for each point takes into account the $95 \%$ confidence interval of the Voigt fit and the uncertainty in the pressure measurement. After plotting the pressure broadening rates, $\Delta v_{\text {pres, }}$ against the system pressure, the slope of the (non-weighted) linear fit of the different points gives the pressure broadening coefficient $\nu_{\text {pres }}(T)$, which is a physical constant proportional to the collisional cross-section of the two colliding species at a certain temperature.

\section{RESULTS}

\section{A. System performance}

A spectrogram, shown in Figure 3, was recorded to examine the spectral purity of the excitation pulse, and the frequency response of the system, using configuration 1. 


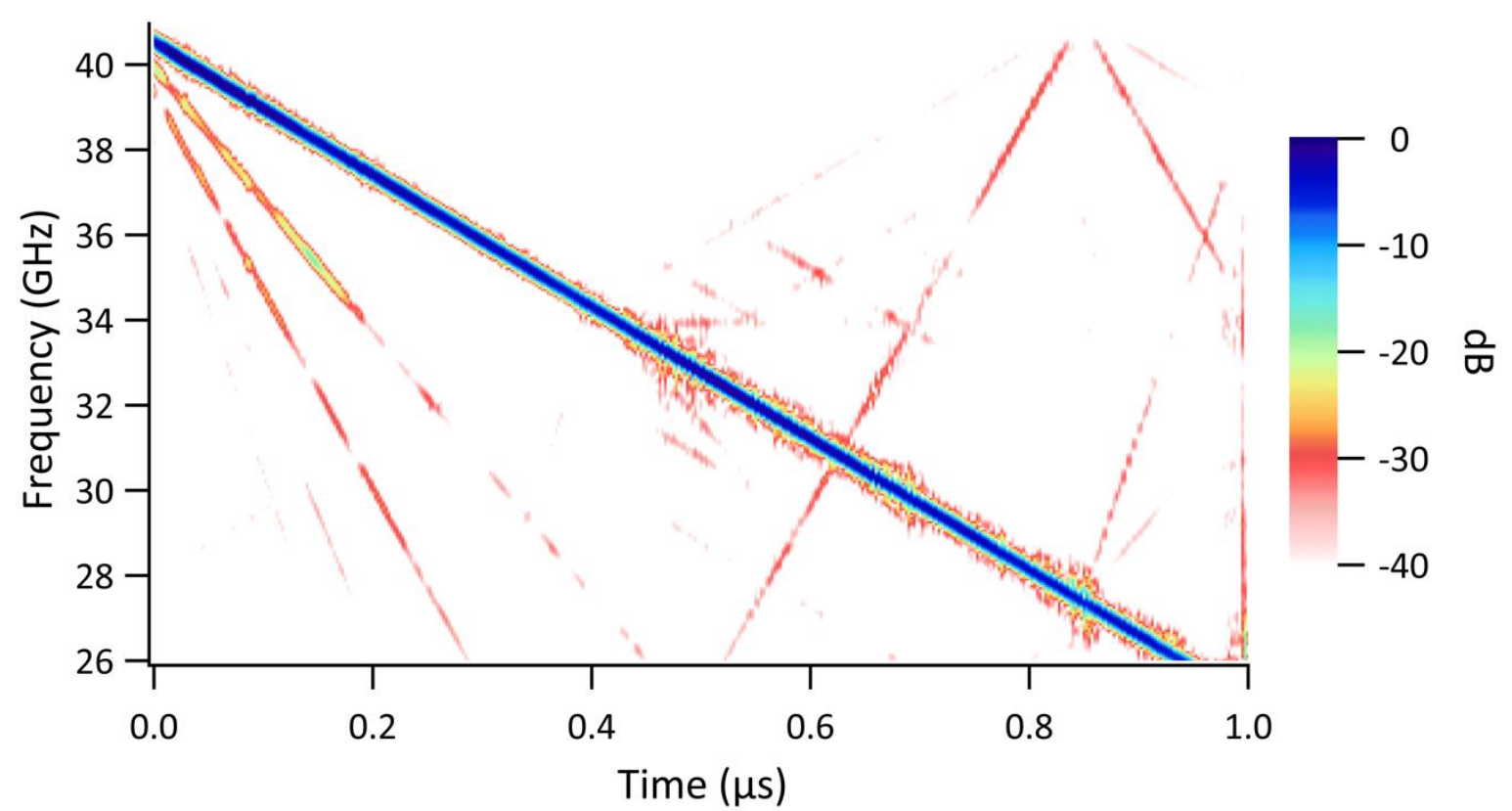

Figure 3. A spectrogram of a chirped pulse from $40.6 \mathrm{GHz}$ to $25.4 \mathrm{GHz}$ over a time of $1 \mu \mathrm{s}$. The most intense point is set arbitrarily to $0 \mathrm{~dB}$. The signal is recorded after having been upconverted, broadcast from the power amplifier, and then downconverted, but without the use of the two LNAs shown in Figure 1. The time-domain spectrum was recorded by the oscilloscope, and is shown at the bottom of the figure.

The LNAs were removed from the circuit for the recording of the spectrogram, which shows a relatively flat response across the range of the instrument, the difference in power between the most intense frequency $(34.85 \mathrm{GHz})$ and the weakest frequency $(26.5 \mathrm{GHz})$ is $5.5 \mathrm{~dB}$. This figure can be improved by tuning the preamplifier stage of the spectrometer to better suit the lower frequencies, but at the expense of power in the upper frequencies. Harmonics of the carrier frequencies begin to appear at around $-25 \mathrm{~dB}$, but for most frequencies the strength of the harmonics and any spurs is below this level. 


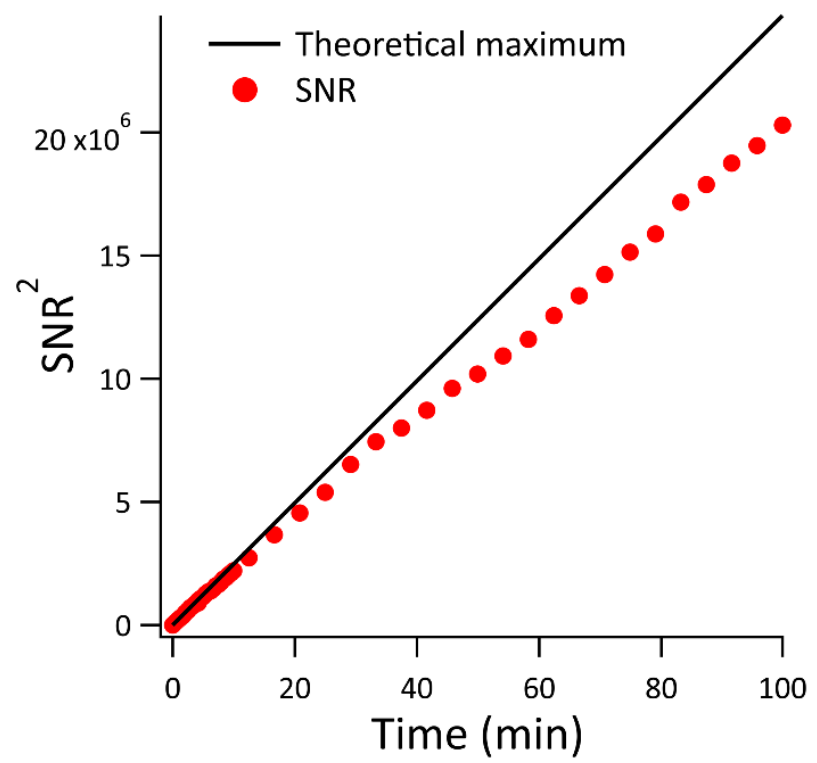

Figure 4. Square of the signal-to-noise ratio (SNR) of an arbitrary single frequency pulse emitted by the broadcast side of the Ka-band chirped pulse Fourier transform spectrometer plot against the time taken to record, at a repetition rate of $1 \mathrm{kHz}$. The black line shows ideal theoretical performance.

Extensive averaging tests were also performed with a range of different instrument configurations to ensure the expected SNR gain with increasing number of averages, extending up to 6 million averages over a 100 minute timescale, as shown in Figure 4. At this limit, the response of the system is $90.6 \%$ of the value expected for a perfectly phase-stable system. Most of the experiments presented here were performed with between 250,000 to 4,000,000 averages with up to 10 minutes in acquisition time. After 250,000 averages, over a time of around 2 minutes, the SNR for the $J=3-2$ transition of 1\% OCS in $9.5 \mu$ bar of He was 5400:1.

The strength of the electrical field generated by the spectrometer was determined via measuring the change in intensity of the FFT of the FID of a transition against the duration of the excitation pulse. ${ }^{38}$ This $^{2}$ produces an oscillating plot known as the Rabi cycle, defined by the following formula:

$$
S \propto e^{\frac{-t_{p}}{T_{2}}}\left|\sin \left(\omega_{r} t_{p}\right)\right|
$$

Where $\omega_{\mathrm{r}}$ is the Rabi frequency, $t_{p}$ is the length of the pulse, and $T_{2}$ is the decay time of the FID. The frequency of the cycle depends only on the transition dipole moment and the electric field strength in the relation:

$$
\omega_{r}=\frac{\mu_{i j} \varepsilon(\omega)}{\hbar}
$$

Where $\mu_{i j}$ is the transition dipole moment, $\hbar$ is the reduced Planck's constant, and $\varepsilon(\omega)$ is the electric field strength at the transition frequency. The transition dipole moments were extracted from the CDMS by dividing the square-root of the line strength factor $\left({ }^{x} S_{i j}\right)$ by the square-root of the lower state degeneracy $\left(g^{\prime \prime}\right)$ and multiplying this value by the dipole moment along the molecular axis $\left(\mu_{x}\right) .^{39}$ 
Table I shows the field strength determined via this method for a range of different transitions, along with the average value over the length of cell using the formula for field intensity at a certain distance given amplifier power and antenna gain:

$$
\text { Ave }=\frac{1}{x_{2}-x_{1}} \int_{x_{1}}^{x_{2}} \frac{\sqrt{30 P 10^{G / 10}}}{x} d x
$$

Where $x_{1}$ is the distance of the start of the flow cell from the antenna, $x_{2}$ is the distance of the end of the flow cell from the antenna, $P$ is the power of the broadcast amplifier in Watts, and $G$ is the gain of the broadcast antenna. There is a distance of $0.25 \mathrm{~m}$ from the output of the amplifier to the start of the molecular sample; the calculation neglects any loss from the cell window. An example Rabi cycle is shown in Figure 5, which shows the intensity of the $J=3-2$ transition of $5.5 \mu$ bar of OCS within the flow cell for a range of different excitation pulse lengths. The output power of the amplifier, and the gain of the horn antenna, vary across the Ka-band, thus it is important to measure the field strength for a range of frequencies in order to accurately determine relative abundance of different molecules.

Table I. The electrical field strength within the flow cell as determined using a range of different methods. The uncertainty given is the $95 \%$ confidence interval for the fit of the Rabi frequency to the experimental data.

\begin{tabular}{lcc}
\hline \multicolumn{1}{c}{ Method of determination } & $\begin{array}{c}\text { Frequency } \\
(\mathrm{GHz})\end{array}$ & $\begin{array}{c}\text { Electric Field Strength } \\
(\mathrm{V} / \mathrm{m})\end{array}$ \\
\hline OCS Rabi cycle & 36.5 & $869 \pm 32$ \\
Vinyl cyanide Rabi cycle & 37.9 & $778 \pm 86$ \\
Benzonitrile Rabi cycle & 36.3 & $826 \pm 49$ \\
Theoretical & 37.0 & 893 \\
\hline
\end{tabular}

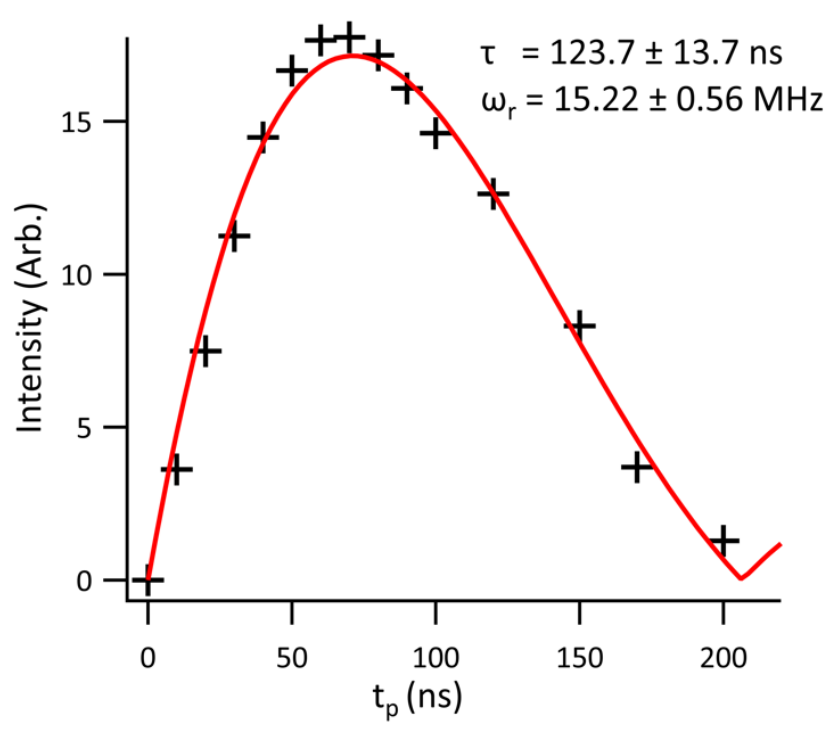


Figure 5. The intensity of the $J=3-2$ transition of OCS plot as a function of excitation duration. Excitation pulses are a single-frequency resonant with the transition energy. The fitting parameters $\tau$ and $\omega_{\mathrm{f}}$ are determined by a least-squares fit of the Rabi cycle formula to the experimental data. The uncertainties given are the $95 \%$ confidence intervals for the fit.

\section{B. Pressure broadening - OCS}

Pressure broadening values for the $J=3-2$ transition of OCS were recorded between $1 \mu$ bar and $32 \mu$ bar. This transition is relatively strong and is the only transition within the range of the spectrometer. This enabled the use of the time-domain Voigt profile for fitting the recorded FIDs. Additionally, the values were reproduced using the frequency-domain Voigt profile fit. Figure 6 shows the plot of pressure broadening against pressure for the two different methods.

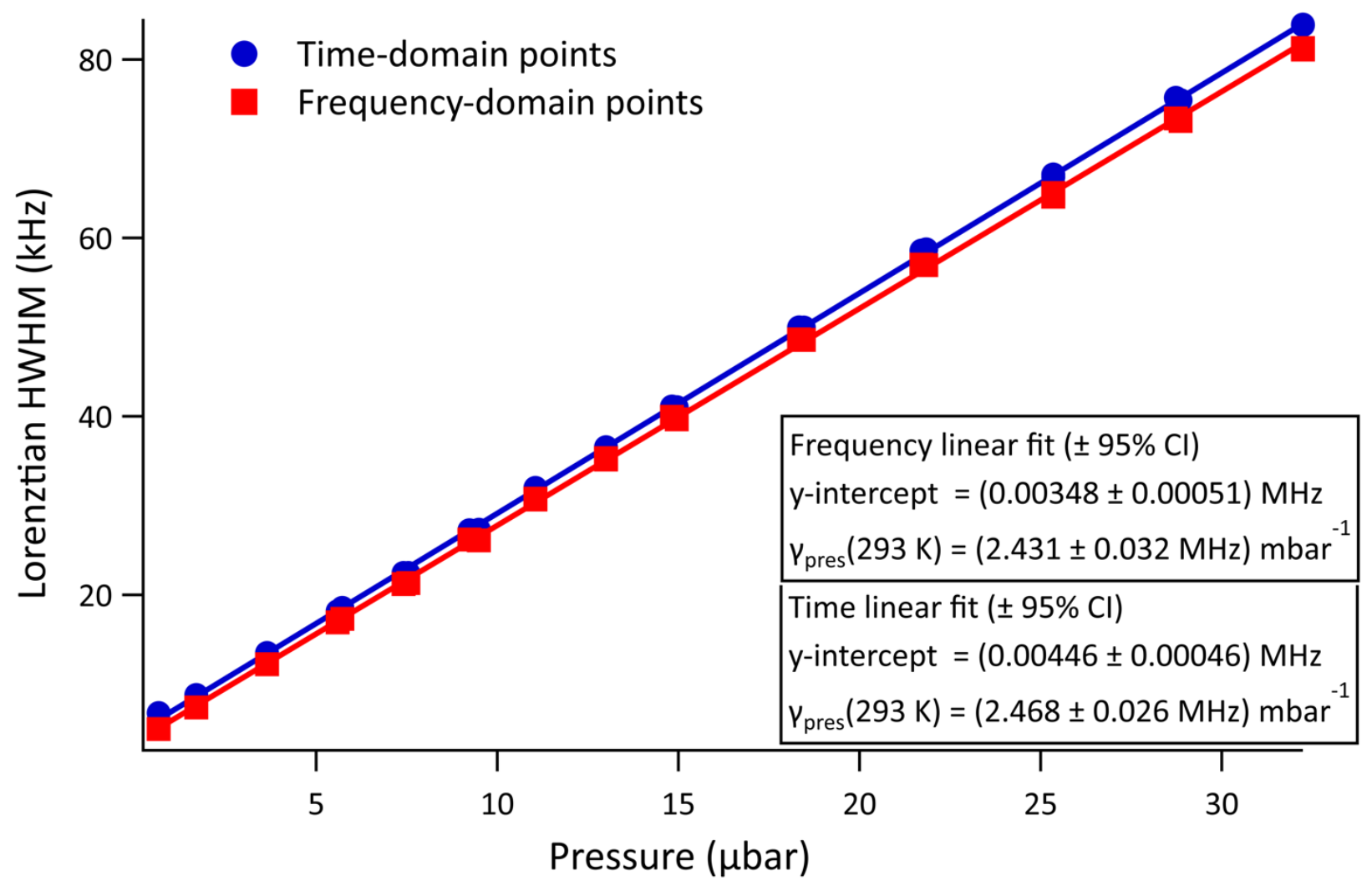

Figure 6. Plot of $\Delta v_{\text {pres }}$ for the fit of the Voigt profile to FIDs of the J=3-2 transition of OCS at different pressure. The data resulting from a fit in the time domain are in blue, data resulting from a fit in the frequency domain are in red. The equations of the linear fits to the two data sets are presented, along with the $95 \%$ confidence interval.

The recorded FIDs were filtered using a correlation filter ${ }^{6}$ and a digital bandpass filter to remove any DC offset and out-of-band noise before a window was selected over the range of the FID for fitting to the timedomain Voigt profile. If the Doppler broadening rate was allowed to float, it returned a value, on average, of 
$(2.834 \pm 0.003) \times 10^{4} \mathrm{~Hz}$ with the uncertainty taken as the $95 \%$ confidence interval. The predicted Doppler broadening for OCS at this frequency is $2.881 \times 10^{4} \mathrm{~Hz}$, which is a difference of around $2 \%$. This would equate to a temperature difference of around $10 \mathrm{~K}$ at room temperature. A similar difference was observed for the transitions in the E-band. ${ }^{6}$ For the pressure broadening values presented here, Doppler broadening was constrained to the theoretical HWHM value for a certain molecule and transition at room temperature, which is given by the formula:

$$
\gamma_{G}=\omega \sqrt{\frac{2 k T \ln 2}{m c^{2}}}
$$

Where $c$ is the speed of light, $\omega$ is the transition frequency, $k$ is the Boltzmann constant, $T$ is the temperature and $m$ is the mass of the species. Constraining Doppler broadening was necessary to improve the fit of FIDs with low SNR. Examples of the two different methods used to fit the FIDs are given in Figure 7.

(a) - Time Domain

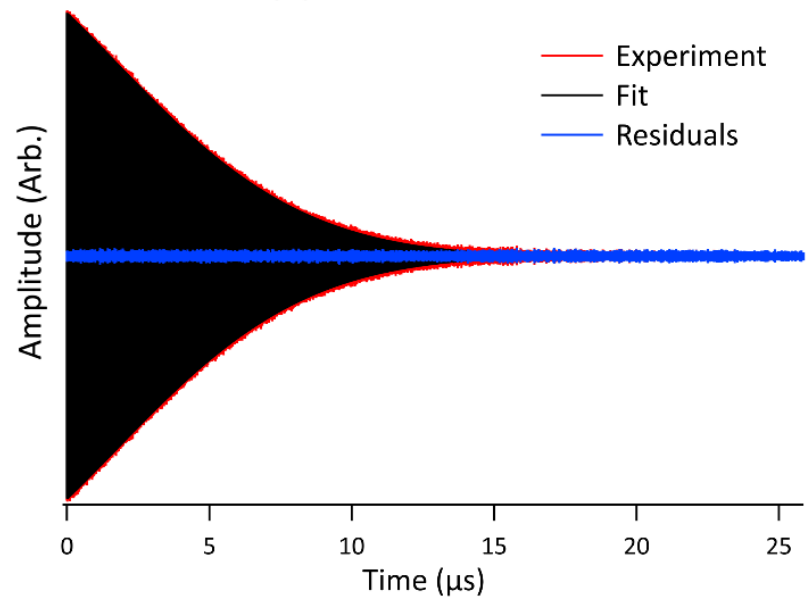

\section{(b) - Frequency Domain}

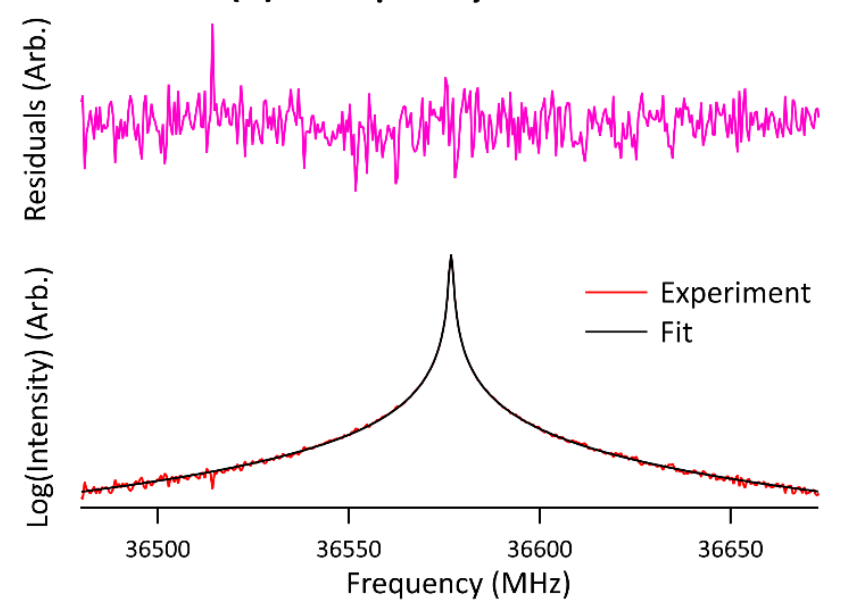

Figure 7. Different displays of fitting results for the $J=3-2$ transition of OCS in a helium buffer gas. Part (a) shows the time-domain fit, with residuals, of a Voigt profile to the recorded FID. Part (b) shows the FFT of the time-domain experimental data, along with a fit in the frequency domain to this data with the accompanying residuals.

The pressure broadening coefficient at $293 \mathrm{~K}$ for the $J=3-2$ transition of OCS was found to be $(2.47 \pm 0.01)$ $\mathrm{MHz} \mathrm{mbar}^{-1}$ with the time-domain fitting, and $(2.43 \pm 0.02) \mathrm{MHz} \mathrm{mbar}^{-1}$ with the frequency-domain fitting.

\section{Pressure broadening - vinyl cyanide}

The Ka-band covers two sets of strong transitions of vinyl cyanide. A fixed amount of the vinyl cyanide/He mixture was introduced into the flow cell, and FIDs recorded for a range of pressures corresponding to increasing flow rates of pure helium. A transition from each set was chosen based on its isolation within the spectrum: $J_{K a, K c}=3_{1,3}-2_{1,2}$ and $J_{K a, K c}=4_{1,4}-3_{1,3}$, and the FIDs from the excitation of these transitions were fit using the time-domain Voigt profile and the frequency-domain Voigt profile. Doppler broadening was constrained 
to the theoretical value at room temperature. The plot of the pressure broadening rates against pressure is shown in Figure 8.
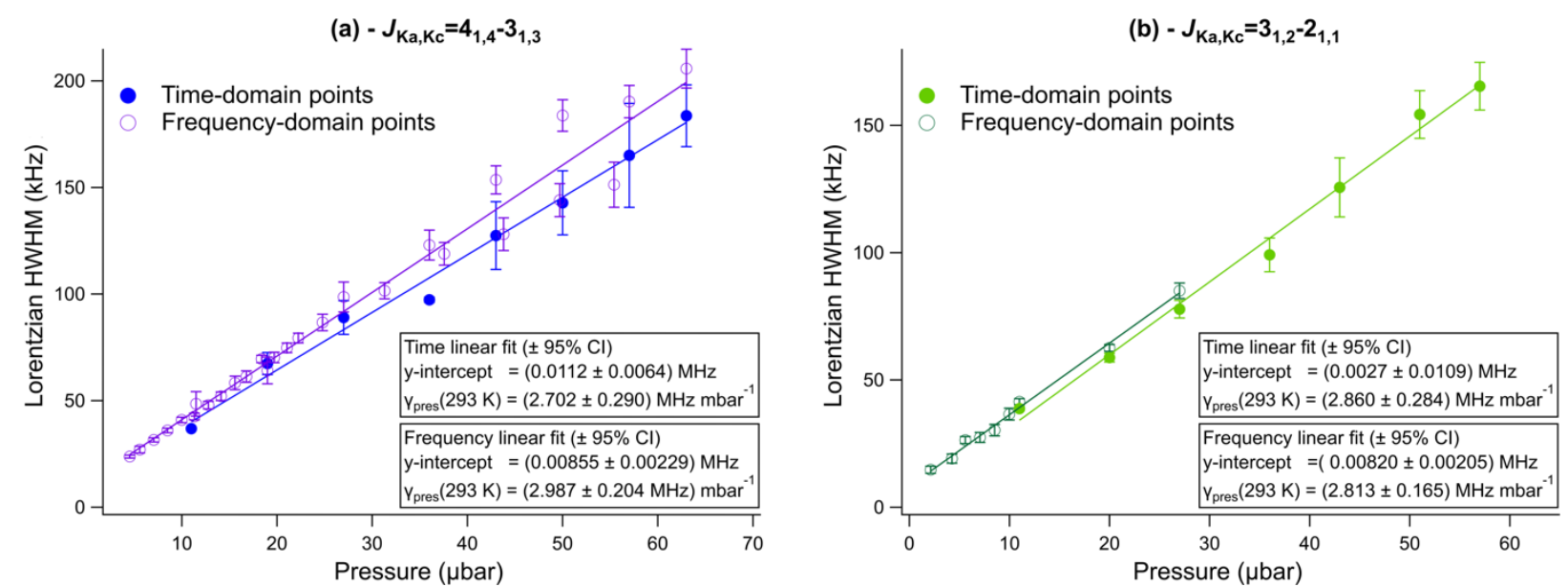

Figure 8. Plots of the HWHM of the Lorentzian component of the Voigt profile fit to FIDs of two different transitions of vinyl cyanide at different pressures, using both the time fitting technique and the frequency fitting technique. Part (a) shows the pressure broadening of the $J_{K a, K c}=4_{1,4}-3_{1,3}$ transition, part (b) shows the broadening of the $J_{K a, K c}=3_{1,2}-2_{1,1}$ transition. The equations for the linear fits to each data set are also displayed.

The pressure broadening coefficients that were determined through each method for the two transitions shown in Figure 8 are displayed in Table II, along with the pressure broadening constants for a number of other transitions of vinyl cyanide determined through the frequency fitting method.

Table II. Pressure broadening coefficients for collisions with He of different transitions of vinyl cyanide as determined through different methods. Uncertainties are taken as the $95 \%$ confidence interval for the linear fit to pressure broadening data. Separate vibrational levels are highlighted.

\begin{tabular}{|c|c|c|c|c|}
\hline $\begin{array}{l}\text { Vibrational } \\
\text { level }\end{array}$ & $\begin{array}{l}\text { Transition } \\
\text { quantum } \\
\text { numbers }\left(J_{K a, k c}\right)\end{array}$ & $\begin{array}{l}\text { Frequency } \\
(\mathrm{MHz})^{\mathrm{a}}\end{array}$ & $\begin{array}{l}Y_{\text {pres }}(293 \mathrm{~K}) \\
\text { from time fit } \\
\left(\mathrm{MHz} \mathrm{mbar}^{-1}\right)\end{array}$ & $\begin{array}{l}V_{\text {pres }}(293 \mathrm{~K}) \text { from } \\
\text { frequency fit } \\
\left(\mathrm{MHz} \mathrm{mbar}^{-1}\right)\end{array}$ \\
\hline$v=0$ & $3_{1,3}-2_{1,2}$ & 27767.4 & & $3.03 \pm 0.19$ \\
\hline$v=0$ & $3_{0,3}-2_{0,2}$ & 28441.0 & & $3.30 \pm 0.34$ \\
\hline$v=0$ & $3_{2,2}-2_{2,1}$ & 28456.9 & & $3.37 \pm 0.21$ \\
\hline$v=0$ & $3_{2,1}-2_{2,0}$ & 28470.8 & & $2.87 \pm 0.27$ \\
\hline$v=0$ & $3_{1,2}-2_{1,1}$ & 29139.1 & $2.86 \pm 0.28$ & $2.81 \pm 0.17$ \\
\hline$v_{11}=1$ & $3_{1,3}-2_{1,2}$ & 27833.2 & & $2.76 \pm 0.41$ \\
\hline$v_{11}=1$ & $3_{1,2}-2_{1,1}$ & 29239.2 & & $3.13 \pm 0.31$ \\
\hline$v=0$ & $4_{1,4}-3_{1,3}$ & 37018.9 & $2.70 \pm 0.29$ & $2.99 \pm 0.20$ \\
\hline
\end{tabular}




\begin{tabular}{llll}
\hline $\boldsymbol{v}=\mathbf{0}$ & $4_{0,4}-3_{0,3}$ & 37904.8 & $2.94 \pm 0.10$ \\
$\boldsymbol{v}=\mathbf{0}$ & $4_{2,3}-3_{2,2}$ & 37939.6 & $3.10 \pm 0.06$ \\
$\boldsymbol{v}=\mathbf{0}$ & $4_{2,2}-3_{2,1}$ & 37974.4 & $3.22 \pm 0.06$ \\
$\boldsymbol{v}=\mathbf{0}$ & $4_{1,3}-3_{1,2}$ & 38847.7 & $3.05 \pm 0.15$ \\
$\boldsymbol{v}_{\mathbf{1 1}}=\mathbf{1}$ & $4_{1,4}-3_{1,3}$ & 37106.4 & $2.59 \pm 0.27$ \\
$\boldsymbol{v}_{\mathbf{1 1}}=\mathbf{1}$ & $4_{2,2}-3_{2,1}$ & 38087.2 & $3.11 \pm 0.27$ \\
$\boldsymbol{v}_{\mathbf{1 1}}=\mathbf{2}$ & $4_{1,4}-3_{1,3}$ & $37192.0^{\mathrm{b}}$ & $3.08 \pm 0.37$ \\
\hline
\end{tabular}

${ }^{a}$ Frequencies taken from the $\mathrm{CDMS}^{39}$ unless otherwise stated.

${ }^{\mathrm{b}}$ Frequency determined from work by Kisiel et al. ${ }^{40}$

\section{Pressure broadening - benzonitrile}

Benzonitrile has many transitions within the range of the Ka-band, which complicates the determination of pressure-broadening parameters. The lack of intensity and the presence of many lines within a single record, even for single-frequency pulses, meant that time-domain fitting was infeasible. However, since most transitions are well separated in frequency, adequate fitting was able to be achieved in the frequency domain. Firstly, self-broadening coefficients were determined for 3 different J-levels; knowing the selfbroadening coefficient helps determine the extent of self-broadening occurring in the helium buffer experiments. Even though the concentration of benzonitrile is below 1\% of He for those experiments, the interactions at this level could still be significant compared to benzonitrile-He collisions. For the $J_{K a, K c}=15_{2,14^{-}}$ $14_{2,13}$ transition, the self-pressure broadening coefficient was determined to be $(57.5 \pm 0.8 \mathrm{MHz}) \mathrm{mbar}^{-1}$. Given a partial pressure of $4.1 \times 10^{-5} \mathrm{mbar}$ of benzonitrile, the estimated contribution of self-collisions to broadening is $0.0024 \mathrm{MHz}$, which is up to $65 \%$ of the recorded value in these experiments. Knowing the partial pressure of benzonitrile and the self-broadening coefficients, the pressure broadening rates obtained with a helium buffer gas at a certain total pressure can be corrected for the contribution of benzonitrile selfcollisions. The self-broadening rate should not affect the value of the pressure broadening coefficient, but will influence the $y$-intercept of the linear fit of pressure broadening rates to pressure. The pressure broadening rates measured at each pressure are plotted for the $J_{K a, K c}=15_{2,14}-14_{2,13}$ transition in Figure 9 . Table III shows the a number of different pressure broadening coefficients determined through the linear fit of pressure broadening rates to pressures with their uncertainty as the $95 \%$ confidence interval. 


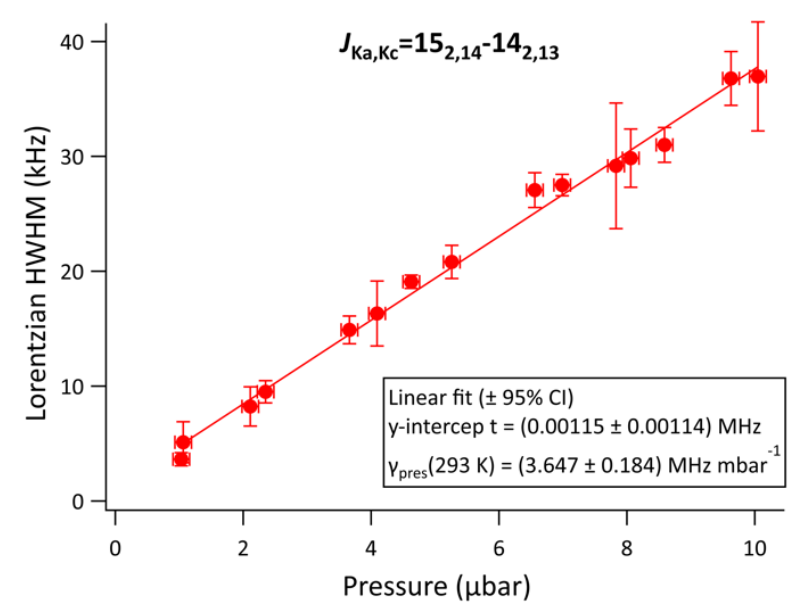

Figure 9. Plot of the HWHM of the Lorentzian component of the Voigt profile fit to the Fourier transform of the FID of the $J_{K a, K c}=15_{2,14-14_{2,13}}$ transition of benzonitrile. The equation of the linear fit to the data is shown.

Table III. Pressure broadening coefficients for various transitions of benzonitrile with two different collisional systems. Uncertainties are taken as the $95 \%$ confidence interval for the linear fit to pressure broadening data.

\begin{tabular}{|c|c|c|c|}
\hline $\begin{array}{l}\text { Transition } \\
\left(J_{K a, K c}\right)\end{array}$ & $\begin{array}{l}\text { Frequency } \\
(\mathrm{MHz})^{\mathrm{a}}\end{array}$ & $\begin{array}{l}\text { Self } Y_{\text {pres }}(293 \mathrm{~K}) \\
\left(\mathrm{MHz} \mathrm{mbar}^{-1}\right)\end{array}$ & $\begin{array}{l}\text { He } \gamma_{\text {pres }}(293 \mathrm{~K}) \\
\left(\mathrm{MHz} \mathrm{mbar}^{-1}\right)\end{array}$ \\
\hline $13_{2,12}-12_{2,11}$ & 34864.9 & & $3.29 \pm 0.71$ \\
\hline $12_{2,10-11_{2,9}}$ & 34898.8 & & $4.02 \pm 0.70$ \\
\hline $14_{0,14}-13_{0,13}$ & 35249.2 & $61.2 \pm 2.2$ & $3.23 \pm 0.98$ \\
\hline $15_{2,14}-14_{2,13}$ & 39897.9 & $57.5 \pm 0.8$ & $3.65 \pm 0.18$ \\
\hline $16_{0,16}-15_{0,15}$ & 40090.9 & & $5.25 \pm 0.21$ \\
\hline
\end{tabular}

${ }^{a}$ Frequencies taken from the $\mathrm{CDMS}^{39}$

\section{E. CRESU flow}

The rotational spectrum of vinyl cyanide over the Ka-band was recorded in a cold CRESU flow at $18 \mathrm{~K}$ and is shown in Figure 10. It is possible to determine the temperature of the probed sample using intensity of these transitions. 


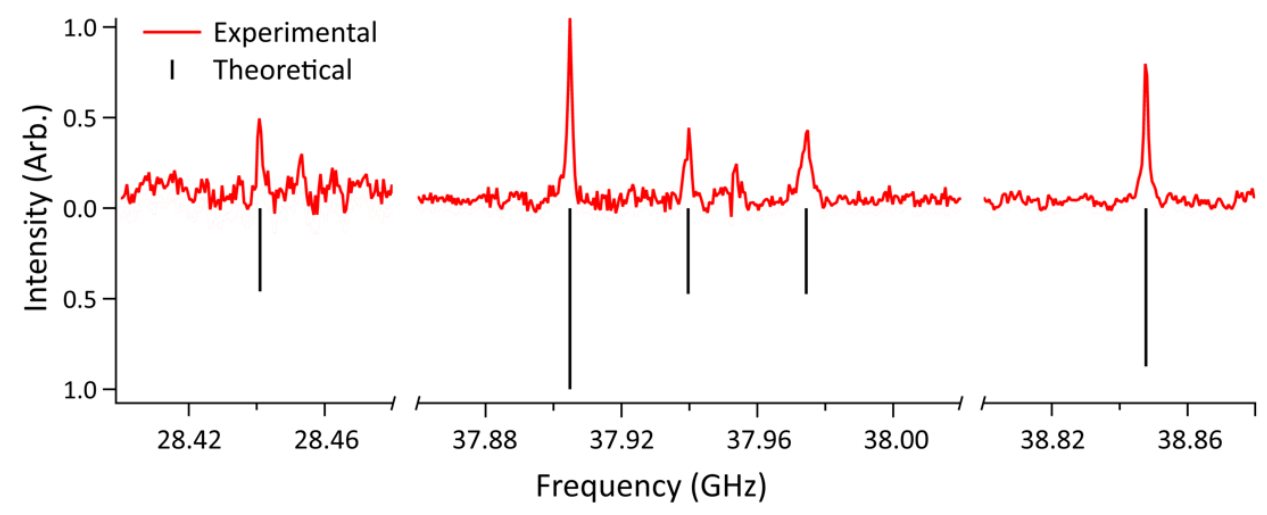

Figure 10. Rotational spectrum of vinyl cyanide in a cold CRESU flow of $18.7 \mathrm{~K}$ recorded using a Ka-band CP-FTMW spectrometer. Each section of the spectrum was probed individually with a single frequency pulse of $10 \mathrm{~ns}$. The spectrum displayed is the magnitude of the Fourier transform of the recorded signals, normalized to the most intense peak. The experimental spectrum is compared to theoretical transition frequencies and intensities at $18.75 \mathrm{~K}$ taken from the $\mathrm{CDMS}^{39,41}$.

The recorded transitions were fit to the Voigt function, in the same way as for the frequency-domain pressure broadening data, in order to give an integrated intensity for each transition. For the CRESU data, the Gaussian broadening was expected to be around 2 orders of magnitude less than the Lorentzian broadening, thus the Gaussian width does not have much influence on the quality of the fit, and was fixed to the values for a vinyl cyanide molecule at $20 \mathrm{~K}$ and the particular frequency of each transition. The hyperfine contributions were neglected in this fit as the widths of the peaks were much larger than the splittings for each transition. The integrated intensity from these measurements is proportional to the intensity of a molecular emission resulting from a single-frequency excitation pulse ${ }^{7,42}$ :

$$
\Delta S=\frac{-\pi \beta(\omega) \omega l}{c} \mu_{i j} \varepsilon(\omega) \Delta N_{0} \sin \left(\frac{\mu_{i j} \varepsilon(\omega)}{\hbar} t_{1}\right) e^{-\left(t-t_{1}\right) / T_{2}} \cos \left[\Delta \omega_{f}\left(t-t_{1}\right)\right]
$$

Where $\Delta S$ is the integrated intensity, $\omega$ is the transition frequency, $\Delta \omega_{f}$ is the detuning of the excitation pulse from the transition frequency, $/$ is the sample path length, $b(\omega)$ is a scaling factor for the response of the receiver at the transition frequency, $t_{1}$ is the pulse duration, $t$ is time the signal is recorded, $\Delta N_{o}$ is the initial population difference and $T_{2}=1 /\left(2 * \pi * \Delta v_{\text {pres }}\right)$ is the decay time of the FID. Since all of the factors contributing to the signal intensity are known for the experiment, save for the population difference $\left(\Delta N_{0}\right)$ between the lower state and upper state levels, $\Delta S$ can be adjusted to give an intensity value solely dependent on temperature: $I(T)$. The temperature dependence of $I(T)$ is given by the equation:

$$
I(T)=a_{2}\left(e^{\frac{-E^{\prime \prime}}{k T}}-e^{\frac{-E^{\prime}}{k T}}\right)
$$

where $I(T)$ is the integrated intensity after adjusting for the extra terms in Eq. 6 besides the population difference, $a_{2}$ is a scaling constant, and $E^{\prime \prime}$ and $E^{\prime}$ are the energies of the lower and upper states respectively. The differential evolution method ${ }^{43}$ was used through the Lmfit Python package ${ }^{37}$ to find the global 
minimum for the sum of the squares of the differences between Eq. 6 and the adjusted integrated intensities from the experiment, weighted by the inverse variance. The results of this method were then used as the first guess for a least squares optimization of the same function, to confirm the values at the minimum. Through this method, the fitting parameters were determined to be: $T=(24 \pm 11) \mathrm{K}$, and $a_{2}=15 \pm 5$ with the uncertainties as the $95 \%$ confidence interval. The plot of the adjusted intensities along with the fit values is show in Figure 11.

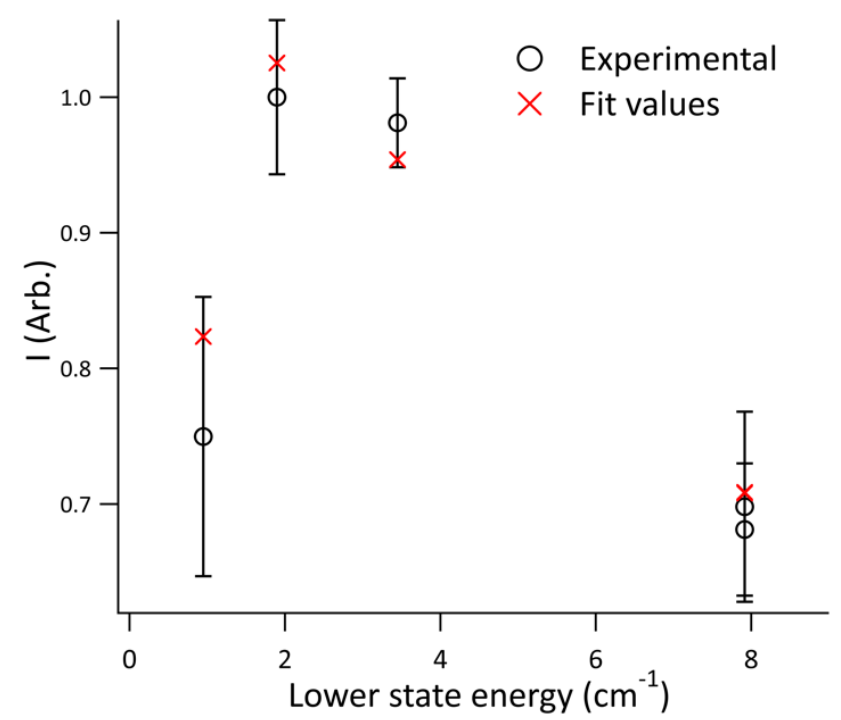

Figure 11. Plot of the intensities of 6 transitions of vinyl cyanide measured in a flow from a Laval nozzle. Also shown are the values determined through fitting the experimental values to the equation for the population difference. The fitting procedure determined a temperature of $(24 \pm 11) \mathrm{K}$ for the experiment, with the uncertainty the $95 \%$ confidence interval.

In order to determine if this rotational temperature is reasonable, a composite temperature due to the contribution of the cold flow, the boundary layer, and the ambient gas to final recorded signal can be estimated given the conditions of the experiment. The boundary layer, which is the region between the cold core and room-temperature gas, and the ambient gas between the broadcast and receiver antennae, can be modelled using the CFD simulation of the flow through the Laval nozzle. The variation of temperature and pressure over the axial distance from the center of the flow core to the position of the spectrometer horns is shown in Figure 12. The low-temperature cold core extends for around $0.3 \mathrm{~cm}$ before rising in temperature through the boundary layer to reach ambient conditions. An important consideration is that pressure broadening coefficients are temperature dependent, and can be roughly modelled by an empirical scaling equation:

$$
\gamma_{\text {pres }}(\mathrm{T})=\gamma_{\text {pres }}(293 \mathrm{~K}) \cdot\left(\frac{T}{293 K}\right)^{-n}
$$


Where $\gamma_{\text {pres }}(293 \mathrm{~K})$ is the pressure broadening rate at $293 \mathrm{~K}, T$ is the temperature and $n$ is a dimensionless value, normally between 0.8 and 0.5 , but yet to be determined for vinyl cyanide. Some typical values for $n$ for collisions with $\mathrm{He}$ are $0.634 \pm 0.025$ for $\mathrm{CH}_{3} \mathrm{CN},{ }^{21}$ and two values for $\mathrm{HC}_{3} \mathrm{~N}$ of $0.49 \pm 0.04$ for $\mathrm{J}=11-10,{ }^{23}$ and $0.717 \pm 0.027$ for $J=24-23 .{ }^{22}$ Based on these results, it was assumed that $n$ lies between 0.5 and 0.7 for vinyl cyanide, and that pressure broadening follows the given equation down to $18 \mathrm{~K}$. The distance of $7.5 \mathrm{~cm}$ between the center of the flow and the antenna was divided into 900 points. In order to determine the relative contribution of each point, a volume was determined. For the first point, the volume was a cylinder with radius $=7.5 / 900 \mathrm{~cm}$ and a length equal to the length of the spectrometer horns $(2.74 \mathrm{~cm})$. For each subsequent point, the volume was calculated to be a hollow cylindrical shell enveloping the earlier points. For the points which generated a shell larger than the height of the spectrometer horns $(2.19 \mathrm{~cm})$, the top and bottom of the cylinder was disregarded. For each point, with a given volume, temperature and pressure, the relative intensity of the $J_{K a, K c}=4_{0,4}-3_{0,3}$ transition of vinyl cyanide was calculated by solving the optical Bloch equations as defined by McGurk et al. for the given conditions. ${ }^{7}$ The optical Bloch equations have been well-examined in previous CP-FTMW spectroscopy papers, and the calculation for signal intensity follows previously establish formalism, so it will not be further discussed here. ${ }^{11,38}$ The calculated intensities are plot as a function of radial distance from the flow core in Figure 12. For each point, the calculated relative intensity of the transition was used as a weighting factor in the determination of the weighted-average temperature for the 900 points, this gave an estimated composite temperature of $(41 \pm 10) \mathrm{K}$. 


\section{(a) - 3D representation of probed space}

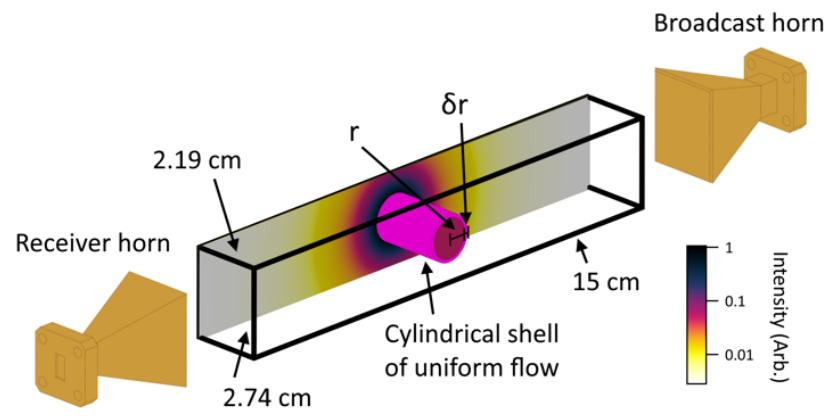

(b) - radial flow properties

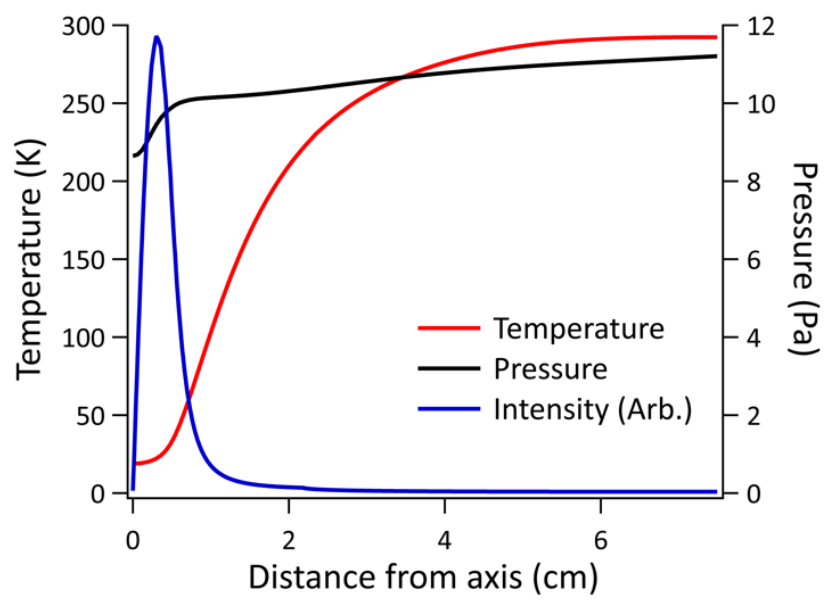

Figure 12. Part (a) shows the isometric representation of the probed space for the CRESU measurements. The rectangular dimensions $(2.74 \mathrm{~cm} \times 2.19 \mathrm{~cm})$ of the two antennae are considered to form a rectangular prism between them, at the center of which is the cold core of the CRESU flow. The electric field is assumed to be uniform throughout this volume. In order to determine the composite rotational temperature that could be expected to be observed from probing this region, the relative intensity of the $J_{\mathrm{Ka}, \mathrm{Kc}}=4_{0,4}-3_{0,3}$ transition of vinyl cyanide was calculated by solving the optical Bloch equations for a series of cylindrical shells of inner, hollow radius $r$ and outer, solid radius $r+\delta r$. The pressure and temperature conditions for each shell were taken from a simulation performed with the OpenFOAM software and the RhoCentralFoam solver. These conditions are shown in part (b) as they vary from the cold core to the ambient conditions near the output of the antennae, along with the resulting intensity calculation. Cylindrical shells which extend beyond the bounds of the rectangular prism were truncated. Part (b) shows that the greatest contribution to the measured signal is expected to originate from the cold core region, which extends to around $0.3 \mathrm{~cm}$ in this experiment.

\section{DISCUSSION}

\section{A. System performance}

Despite the use of an absorbent material within the flow cell, reflections from the excitation pulse lasted up to $400 \mathrm{~ns}$ after the end of the pulse. These signals could originate from reflections between the broadcast 
and receiver horns, or come from the electrical path of the receiver, due to standing waves arising from slight mismatches in impedance between the different components. Given the fast disappearance of the FID, a large gain in SNR can be made by reducing the length of any unwanted transient signals in the spectrometer. The electric field strength as determined by the Rabi cycle plots is slightly less than that predicted simply using the output power of the amplifier and the gain of the broadcast horn. This is likely due to losses from the Teflon window, and absorbance of the beam by the Eccosorb within the flow cell. Park et al. observe a similar reduction in power from basic estimations. ${ }^{38}$

The effective range of the spectrometer can be extended slightly outside of the Ka-band, down to 26.0 GHz and up to $42 \mathrm{GHz}$, with less than $3 \mathrm{~dB}$ loss in power. The response at different frequencies varies across the spectral range, and the addition of the LNAs creates extra fluctuations on top of those shown in the spectrogram in Figure 1. Due to this, careful tuning of the spectrometer is required to obtain optimal response at each frequency. The performance of the spectrometer with high levels of averaging depends upon the phase-stability of the various components involved. At 6 million averages, there is a loss of about $6 \%$ of the expected signal given perfect phase-coherence. This is acceptable for these experiments, but does show that there can be further improvements in SNR with more stable components. The spectrometer was designed so that the same LO source is used for both the upconversion and downconversion stages, this eliminates phase-noise introduced by the LO source. Consequently, no change in performance was observed when different LO sources, such as a PDRO or a synthesizer, were used. Another arrangement of the spectrometer has been tested using a direct-digital synthesis (DDS) card as the pulse source. ${ }^{44}$ This requires adjustments in the timing of the system compared to the configurations shown in figure 1. Despite this, averaging performance is still decent to 6 million averages; in this case the measured loss in SNR is about $10 \%$ compared to a perfectly phase-stable system.

\section{B. Pressure broadening}

Pressure broadening values are not precisely determined for many molecules, but OCS is a chief exception, with measurements performed using a range of different methods including Stark switching experiments, klystron absorption experiments, and maser experiments. The results of the studies that determined pressure broadening coefficients for the OCS-He collisional system at room temperature are shown in Table IV.

Table IV. Pressure broadening coefficients at room temperature for a range of different transitions of OCS in a helium buffer environment. Uncertainty for the value reported from this work is taken to be the $95 \%$ confidence interval of the linear fit to pressure broadening data.

OCS transition $\quad V_{\text {pres }}$ at room temperature




\begin{tabular}{lr}
\hline \multicolumn{2}{c}{$\left(\mathrm{MHz} \mathrm{mbar}^{-1}\right)$} \\
\hline$J=1-\mathbf{0}^{\mathrm{a}}$ & $2.45 \pm 0.04$ \\
$J=3-\mathbf{2}^{\mathrm{b}}$ & $2.5 \pm 0.1$ \\
$J=3-\mathbf{2}^{\mathrm{c}}$ & $2.43 \pm 0.03$ \\
$J=5-4^{\mathrm{d}}$ & $2.39 \pm 0.01$ \\
$J=5-6\left(\text { P branch, } \mathbf{V}_{3}\right)^{\mathrm{e}}$ & $2.6 \pm 0.2$ \\
$J=6-5^{\text {d }}$ & $2.37 \pm 0.01$ \\
$J=7-6^{\text {d }}$ & $2.38 \pm 0.01$ \\
$J=11-10\left(R \text { branch, } \mathbf{v}_{3}\right)^{\mathrm{e}}$ & $2.2 \pm 0.2$ \\
\hline
\end{tabular}

${ }^{a}$ From Ref. 15

${ }^{b}$ From Ref. 14

c From this work

d From Ref. 6

e From Ref. 17

The value determined for $J=3-2$ via frequency-domain Voigt fitting of FIDs fits within the uncertainty of the study by Story et al. on the same transition. ${ }^{14}$ Pressure broadening coefficients, in general when perturbed by a rare-gas, tend to fall from $J=1-0$ as the $J$-level increases, as observed here for OCS-He. The time-domain pressure broadening rates for OCS-He are consistently slightly higher than the frequency-domain data. This could be due to the effect of resonant reflections within the system, which also decay over time and are non-pressure dependent but would be separated from the FID in the frequency space whereas they could overlap with the FID in the time domain. For cases with good SNR, both methods produce accurate results, however at high pressures with a low SNR the time-domain fitting method was still able to give reasonable results in cases where frequency-domain fitting failed. Frequency-domain fitting was required for cases where multiple transitions were present within the same recorded FID yet were separated in the frequency domain.

Measurements of pressure broadening coefficients have not been performed before for vinyl cyanide for any collisional partner, despite recent interest in relation to its detection in the atmosphere of Titan. ${ }^{45}$ There is no evidence of any dependence of pressure broadening on the different $K_{a}$ and $K_{c}$ levels of these transitions at the precision attained in this experiment. $K$-levels should not have an observable effect for the case of $\mathrm{He}$ as the perturbing collider, although $\mathrm{K}$-dependence in the case of larger colliders such as $\mathrm{N}_{2}$ has been observed. ${ }^{21}$ The different vibrational levels were also found to have little impact on pressure broadening coefficients beyond the uncertainty of these measurements, however, previous studies have found small differences between vibrational levels. ${ }^{46}$ Despite rapid rotational cooling in CRESU experiments, 
the vibrational temperature remains relatively constant, thus knowledge of pressure broadening parameters of different vibrational states could be important for future studies.

A comparison can be made of the pressure broadening coefficients of a few cyanide-containing molecules, which is shown in Table $V$.

Table V. Pressure broadening coefficients for related molecules for a range of different transitions with He as the collider.

\begin{tabular}{|c|c|}
\hline Species (transition) & $\begin{array}{c}V_{\text {pres }} \text { at room temperature } \\
\left(\mathrm{MHz} \mathrm{mbar}^{-1}\right)\end{array}$ \\
\hline $\mathrm{HCN}(J=1-0)^{\mathrm{a}}$ & $1.51 \pm 0.02$ \\
\hline $\mathrm{CH}_{3} \mathrm{CN}(J=0-1 K=0, J=1-2 K= \pm 1)^{\mathrm{b}}$ & $2.57 \pm 0.03,2.70 \pm 0.02$ \\
\hline $\mathrm{HC}_{3} \mathrm{~N}(J=3-2)^{\mathrm{c}}$ & $3.1 \pm 0.1$ \\
\hline $\mathrm{CH}_{2} \mathrm{CHCN}\left(J_{\mathrm{Ka}, \mathrm{Kc}}=3_{1,3}-2_{1,2}, J_{\mathrm{Ka}, \mathrm{Kc}}=4_{1,4}-3_{1,3}\right)^{\mathrm{d}}$ & $3.03 \pm 0.19,3.05 \pm 0.15$ \\
\hline$c-C_{6} \mathrm{H}_{5} \mathrm{CN}\left(J_{\mathrm{Ka}, \mathrm{Kc}}=15_{2,14}-14_{2,13}\right)^{\mathrm{d}}$ & $3.65 \pm 0.18$ \\
\hline
\end{tabular}

${ }^{\text {a }}$ From Ref. 27

${ }^{b}$ From Ref. 20

${ }^{\mathrm{c}}$ From Ref. 23

${ }^{\mathrm{d}}$ From this work

The results fit a general trend of increasing pressure broadening coefficients with increasing molecular size. The coefficients for the transitions of vinyl cyanide are similar to cyanoacetylene $\left(\mathrm{HC}_{3} \mathrm{~N}\right)$, which would be expected given the similarity in size and composition of the two molecules. Looking towards heavier molecules, which are of interest to the CRESUCHIRP project using the Ka-band spectrometer, a significant increase in pressure broadening for benzonitrile compared to the smaller molecules in observed. The concern for studying such a molecule in an environment such as a CRESU flow is the increase in pressure broadening at lower temperatures. For the related molecule fluorobenzene, Patterson and Doyle determined the collisional cross-section with helium to be $2.8 \times 10^{-14} \mathrm{~cm}^{2}$ at $8 \mathrm{~K}$ for the $J_{\mathrm{Ka}, \mathrm{Kc}}=2_{1,1}-3_{1,2}$ transition, which corresponds to a pressure broadening coefficient of $86 \mathrm{MHz} \mathrm{mbar}^{-1}{ }^{47}$ This value is at least 3 times greater than the pressure broadening coefficient for vinyl cyanide at $8 \mathrm{~K}$, implying that benzonitrile would be extremely difficult to detect in a high-pressure CRESU flow.

In the line-shape fitting performed here, different hyperfine levels were assumed to have the same pressure broadening rates within the same transition. Theoretical work by Green has suggested that nuclear spin plays a role in collision dynamics and thus the resulting line-shape will differ from the summed Voigt approach, however, Green suggests that the deviation would be small compared to experimental uncertainties. ${ }^{48}$ Nonetheless, a change in pressure broadening rates for different hyperfine transitions has 
been previously observed, with the effect shown to cause a difference in up to $10 \%$ of the pressure broadening rate. ${ }^{28,49,50}$ Green asserts that the hyperfine spectrum will tend towards the expected shape neglecting hyperfine contributions as the pressure rises, and the pressure broadening rate becomes large compared to the hyperfine splitting. The difference in frequency of hyperfine split transitions in many nitrogen containing molecules is much less than the HWHM at the high pressures used in CRESU experiments, thus, the pressure broadening coefficients reported here can be considered to be associated with a molecule without any hyperfine contribution to its rotational spectrum.

A small non-zero intercept for the linear fit in the pressure broadening data was observed for all molecules. This suggests that there is some other source of broadening for the species besides collisions with helium and Doppler broadening. For example, self-collisions between species of interest would contribute a fixed amount to pressure broadening, as would any other background species within the flow cell. The background pressure in the cell is normally around $10^{-3} \mu \mathrm{bar}$, so collisions with background species such as $\mathrm{H}_{2} \mathrm{O}$ or $\mathrm{N}_{2}$ are likely to be negligible. Also, wall collisions may give a small contribution to the broadening of the transitions, although in this case this effect is most likely negligible. Since these effects are relatively small compared to helium collisions or are fixed for experiments across varying helium pressures, they should not influence the pressure broadening coefficient. Furthermore, misalignments and inhomogeneous fields could have a fixed, small effect on line broadening. Finally, non-Voigt effects such as Dicke-narrowing and the speed-dependence of collisions are likely to be small in the case of a collision between a heavy species and helium, and are not considered in the treatment of the data obtained from this experiment. Figure 7(b) shows that the residuals for a Voigt fit to the experimental line-shape do not miss any contribution to the line-shape above the noise level.

\section{CRESU flow}

The results presented here show preliminary spectra originating from a CPUF experiment. A rudimentary analysis of the spectra through signal intensity and pressure broadening indicate that the spectrometer is probing cold gas. The rotational temperature was determined to be $(24 \pm 11) \mathrm{K}$ by fitting experimental line intensities to their initial population differences, compared to an estimated composite temperature of (41 \pm 10) $\mathrm{K}$ from calculations of signal intensity using certain conditions given through CFD simulations. The calculated approach assumes a value of 0.6 for the pressure broadening temperature dependence powerfactor $n$, and that the rough relation between room-temperature pressure broadening coefficients and lower-temperatures coefficients shown in Eq. 7 holds down to $18 \mathrm{~K}$. At low temperatures (generally below $10 \mathrm{~K}$ ), experimental work shows that pressure broadening rates will deviate from this rough relationship to room temperature rates, reaching a steady value whilst Eq. 7 implies an accelerating rise down to $0 \mathrm{~K} .{ }^{28,51} \mathrm{It}$ is unfortunately not feasible to calculate low-temperature pressure broadening coefficients within the scope 
of CRESU experiments; however, given the decent agreement between the different temperatures, it is evidently not essential to know these coefficients to high accuracy in order to give a good estimate of the composite temperature of the experimental environment.

The CRESU core is cold and dense, while the warmer boundary layers are sparser. This, combined with the change of the partition function over temperature, means that signal from the CRESU core will be stronger than any signal coming from the ambient gas by a factor of around 30 for low energy transitions. In experiments on reaction dynamics, the reaction will be initiated by a laser fired down the length of the CRESU core. This has the advantage that any species generated by this laser pulse will reside within the cold CRESU core, and there will be no contribution from the warmer regions. The main concern for kinetics experiments in CRESU flows is that the high level of pressure broadening will have a severe impact on the detection limit for CP-FTMW spectrometers. This work shows that it is possible to obtain quantitative data on a relatively large molecule (vinyl cyanide) in a continuous CRESU flow; however, larger molecules such as benzonitrile will require experimental adjustments to the traditional CRESU experiment in order to reach an acceptable limit of detection.

\section{CONCLUSION}

A novel Ka-band spectrometer has been built and successfully tested on a number of different molecular species and experimental configurations. Pressure broadening data with a helium collider were determined for OCS, vinyl cyanide and benzonitrile for a range of different transitions in the Ka-band. The data did not show much variation between the different transitions of the molecules studied over this frequency range, but higher precision experiments may show the expected inverse dependence on J-level. The pressure broadening data recorded at room temperature can be extrapolated to determine the feasibility of studies at low temperatures and high pressures within CRESU flows. Vinyl cyanide was introduced into a cold CRESU flow determined to have a cold core of around $18 \mathrm{~K}$ by Pitot tube measurements. The intensities of the vinyl cyanide transitions in cold flow conditions showed that the recorded spectrum is dominated by molecules at the cold conditions. The relative intensities of the transitions agreed well will those determined through calculation. These experiments lay out the basis for performing quantitative, precision experiments in the high-pressure environments common in studies of molecular kinetics.

\section{Acknowledgments}

The authors would like to thank Jonathan Courbe, Jonathan Thiévin, Didier Biet, Ewen Gallou, and Alexandre Dapp for technical support. Luc Briand is also thanked for his programming contributions to the CRESCHIRP experiments. Furthermore, Ilsa Cooke and Divita Gupta are thanked for assistance with some of the 
instrumental construction, and in particular for llsa Cooke, her contribution to the editing of the manuscript. The authors acknowledge funding from the European Research Council (ERC) under the European Union's Horizon 2020 research and innovation programme under grant agreement 695724-CRESUCHIRP. The authors are also grateful for support from the European Regional Development Fund, the Region of Brittany and Rennes Metropole. This work was supported by the French National Programme "Physique et Chimie du Milieu Interstellaire" (PCMI) of CNRS/INSU with INC/INP co-funded by CEA and CNES.

\section{DATA AVAILABILITY STATEMENT}

The data that support the findings of this study are available from the corresponding author upon reasonable request.

\section{REFERENCES}

(1) Cooke, I. R.; Sims, I. R. Experimental Studies of Gas-Phase Reactivity in Relation to Complex Organic Molecules in Star-Forming Regions. ACS Earth Space Chem. 2019, 3 (7), 1109-1134. https://doi.org/10.1021/acsearthspacechem.9b00064.

(2) Potapov, A.; Canosa, A.; Jiménez, E.; Rowe, B. Uniform Supersonic Chemical Reactors: 30 Years of Astrochemical History and Future Challenges. Angew. Chem. Int. Ed. 2017, 56 (30), 8618-8640. https://doi.org/10.1002/anie.201611240.

(3) Brown, G. G.; Dian, B. C.; Douglass, K. O.; Geyer, S. M.; Shipman, S. T.; Pate, B. H. A Broadband Fourier Transform Microwave Spectrometer Based on Chirped Pulse Excitation. Rev. Sci. Instrum. 2008, 79 (5), 053103. https://doi.org/10.1063/1.2919120.

(4) Oldham, J. M.; Abeysekera, C.; Joalland, B.; Zack, L. N.; Prozument, K.; Sims, I. R.; Park, G. B.; Field, R. W.; Suits, A. G. A Chirped-Pulse Fourier-Transform Microwave/Pulsed Uniform Flow Spectrometer. I. The Low-Temperature Flow System. J. Chem. Phys. 2014, 141 (15), 154202. https://doi.org/10.1063/1.4897979.

(5) Abeysekera, C.; Zack, L. N.; Park, G. B.; Joalland, B.; Oldham, J. M.; Prozument, K.; Ariyasingha, N. M.; Sims, I. R.; Field, R. W.; Suits, A. G. A Chirped-Pulse Fourier-Transform Microwave/Pulsed Uniform Flow 
Spectrometer. II. Performance and Applications for Reaction Dynamics. J. Chem. Phys. 2014, 141 (21), 214203. https://doi.org/10.1063/1.4903253.

(6) Hays, B. M.; Guillaume, T.; Hearne, T. S.; Cooke, I. R.; Gupta, D.; Abdelkader Khedaoui, O.; Le Picard, S. D.; Sims, I. R. Design and Performance of an E-Band Chirped Pulse Spectrometer for Kinetics Applications: OCS - He Pressure Broadening. J. Quant. Spectrosc. Radiat. Transf. 2020, 250, 107001. https://doi.org/10.1016/j.jqsrt.2020.107001.

(7) McGurk, J. C.; Schmalz, T. G.; Flygare, W. H. Fast Passage in Rotational Spectroscopy: Theory and Experiment. J. Chem. Phys. 1974, 60 (11), 4181-4188. https://doi.org/10.1063/1.1680886.

(8) Abeysekera, C.; Joalland, B.; Ariyasingha, N.; Zack, L. N.; Sims, I. R.; Field, R. W.; Suits, A. G. Product Branching in the Low Temperature Reaction of CN with Propyne by Chirped-Pulse Microwave Spectroscopy in a Uniform Supersonic Flow. J. Phys. Chem. Lett. 2015, 6 (9), 1599-1604. https://doi.org/10.1021/acs.jpclett.5b00519.

(9) Dias, N.; Joalland, B.; Ariyasingha, N. M.; Suits, A. G.; Broderick, B. M. Direct versus Indirect Photodissociation of Isoxazole from Product Branching: A Chirped-Pulse Fourier Transform Mm-Wave Spectroscopy/Pulsed Uniform Flow Investigation. J. Phys. Chem. A 2018, 122 (38), 7523-7531. https://doi.org/10.1021/acs.jpca.8b04713.

(10) Broderick, B. M.; Suas-David, N.; Dias, N.; Suits, A. G. Isomer-Specific Detection in the UV Photodissociation of the Propargyl Radical by Chirped-Pulse Mm-Wave Spectroscopy in a Pulsed QuasiUniform Flow. Phys. Chem. Chem. Phys. 2018, 20 (8), 5517-5529. https://doi.org/10.1039/C7CP06211G.

(11) Fontanari, D.; Bray, C.; Dhont, G.; Mouret, G.; Cuisset, A.; Hindle, F.; Bocquet, R.; Hickson, K. M. Molecules Probed with a Slow Chirped-Pulse Excitation: Analytical Model of the Free-Induction-Decay Signal. Phys. Rev. A 2019, 100 (4), 043407. https://doi.org/10.1103/PhysRevA.100.043407.

(12) Jefferts, K. B.; Penzias, A. A.; Wilson, R. W.; Solomon, P. M. Detection of Interstellar Carbonyl Sulfide. Astrophys. J. Lett. 1971, 168, L111-L113. https://doi.org/10.1086/180795. 
(13) Bezard, B.; de Bergh, C.; Crisp, D.; Maillard, J.-P. The Deep Atmosphere of Venus Revealed by HighResolution Nightside Spectra. Nature 1990, 345, 508-511. https://doi.org/10.1038/345508a0.

(14) Story, I. C.; Metchnik, V. I.; Parsons, R. W. The Measurement of the Widths and Pressure-Induced Shifts of Microwave Spectra Lines. J. Phys. B At. Mol. Phys. 1971, 4 (4), 593-608. https://doi.org/10.1088/0022-3700/4/4/023.

(15) Mäder, H.; Ekkers, J.; Hoke, W.; Flygare, W. H. A $\pi, \tau, \pi / 2$ Type Pulse Sequence Method for the Determination of T1 in Rotational Transitions. J. Chem. Phys. 1975, 62 (11), 4380-4387. https://doi.org/10.1063/1.430338.

(16) Liuima, F. A.; Bushkovitch, A. V.; Rouse, A. G. Pressure Broadening of OCS in Foreign Gas Mixtures. Phys. Rev. 1954, 96 (2), 434-435. https://doi.org/10.1103/PhysRev.96.434.

(17) Broquier, M.; Picard-Bersellini, A.; Whitaker, B. J.; Green, S. Rotational Inelastic Cross Sections for OcSAr, OCS-He, OCS-H2 Collisions: A Comparison between Theory and Experiment. J. Chem. Phys. 1986, 84 (4), 2104-2107. https://doi.org/10.1063/1.450421.

(18) Gardner, F. F.; Winnewisser, G. The Detection of Interstellar Vinyl Cyanide (Acrylonitrile). Astrophys J Lett 1975, 195 (3), L127-L130.

(19) McGuire, B. A.; Burkhardt, A. M.; Kalenskii, S.; Shingledecker, C. N.; Remijan, A. J.; Herbst, E.; McCarthy, M. C. Detection of the Aromatic Molecule Benzonitrile (c-C6H5CN) in the Interstellar Medium. Science 2018, 359 (6372), 202-205. https://doi.org/10.1126/science.aao4890.

(20) Mäder, H.; Bomsdorf, H.; Andresen, U. The Measurement of Rotational Relaxation Time T2 for CH3C15N Self-and Foreign Gas Collisions. Z. Für Naturforschung A 2014, 34 (7), 850-857. https://doi.org/10.1515/zna-1979-0709.

(21) Colmont, J.-M.; Rohart, F.; Wlodarczak, G.; Bouanich, J.-P. K-Dependence and Temperature Dependence of N2-, H2-, and He-Broadening Coefficients for the J=12-11 Transition of Acetonitrile CH3C14N Located near 220.7GHz. J. Mol. Spectrosc. 2006, 238 (1), 98-107. https://doi.org/10.1016/j.jms.2006.04.017. 
(22) Colmont, J.-M.; Rohart, F.; Wlodarczak, G. N2-, H2-, and He-Induced Collisional Broadening of the J=24 $\leftarrow 23$ Transition of HC3N Located near $218.3 \mathrm{GHz}$ at Different Temperatures. J. Mol. Spectrosc. 2007, 241 (2), 119-123. https://doi.org/10.1016/j.jms.2006.11.007.

(23) Rohart, F. Low-Temperature Dependence of the Foreign Gas Relaxation of HC3N with Microwave Coherent Transients Induced by Frequency Switching. J. Mol. Spectrosc. 1993, 158 (2), 287-297. https://doi.org/10.1006/jmsp.1993.1073.

(24) Mehrotra, S. C.; Dreizler, H.; Mäder, H. Investigations of Self-, H2- and He-Broadening for Rotational Transitions of HCCC15N, CF3D and CF3CCH by the Microwave Transient Emmission Technique. J.

Quant. Spectrosc. Radiat. Transf. 1985, 34 (3), 229-231. https://doi.org/10.1016/0022-4073(85)90003-

2.

(25) Bomsdorf, H.; Mäder, H. Investigation of T1-Relaxation by a Microwave Pulse Technique for HCCC15N Rotational Transitions. Z. Naturforschung Teil A 1984, 39, 1212-1221. https://doi.org/10.1515/zna1984-1212.

(26) Nerf, R. B. Pressure Broadening of the $\mathrm{J}=1 \leftarrow 0$ Transition of Hydrogen Cyanide. J. Mol. Spectrosc. 1975, 58 (3), 479-480. https://doi.org/10.1016/0022-2852(75)90227-1.

(27) Colmont, J. M. Collisional Broadening of the $\mathrm{J}=1 \leftarrow 0$ Transition of HC15N by Nitrogen, Oxygen, Helium, and Air. J. Mol. Spectrosc. 1985, 114 (2), 298-304. https://doi.org/10.1016/00222852(85)90227-9.

(28) Ronningen, T. J.; De Lucia, F. C. Helium Induced Pressure Broadening and Shifting of HCN Hyperfine Transitions between 1.3 and 20 K. J. Chem. Phys. 2005, 122 (18), 184319. https://doi.org/10.1063/1.1895905.

(29) Zaleski, D. P.; Neill, J. L.; Muckle, M. T.; Seifert, N. A.; Brandon Carroll, P.; Widicus Weaver, S. L.; Pate, B. H. A Ka-Band Chirped-Pulse Fourier Transform Microwave Spectrometer. J. Mol. Spectrosc. 2012, 280, 68-76. https://doi.org/10.1016/j.jms.2012.07.014. 
(30) Reinhold, B.; Finneran, I. A.; Shipman, S. T. Room Temperature Chirped-Pulse Fourier Transform Microwave Spectroscopy of Anisole. J. Mol. Spectrosc. 2011, 270 (2), 89-97. https://doi.org/10.1016/j.jms.2011.10.002.

(31) Gupta, D.; Cheikh Sid Ely, S.; Cooke, I. R.; Guillaume, T.; Abdelkader Khedaoui, O.; Hearne, T. S.; Hays, B. M.; Sims, I. R. Low Temperature Kinetics of the Reaction Between Methanol and the CN Radical. J. Phys. Chem. A 2019, 123 (46), 9995-10003. https://doi.org/10.1021/acs.jpca.9b08472.

(32) Greenshields, C. J.; Weller, H. G.; Gasparini, L.; Reese, J. M. Implementation of Semi-Discrete, NonStaggered Central Schemes in a Colocated, Polyhedral, Finite Volume Framework, for High-Speed Viscous Flows. Int. J. Numer. Methods Fluids 2010, 63 (1), 1-21. https://doi.org/10.1002/fld.2069.

(33) Weller, H. G.; Tabor, G.; Jasak, H.; Fureby, C. A Tensorial Approach to Computational Continuum Mechanics Using Object-Oriented Techniques. Comput. Phys. 1998, 12 (6), 620-631. https://doi.org/10.1063/1.168744.

(34) Park, G. B.; Field, R. W. Perspective: The First Ten Years of Broadband Chirped Pulse Fourier Transform Microwave Spectroscopy. J. Chem. Phys. 2016, 144 (20), 200901. https://doi.org/10.1063/1.4952762.

(35) Steber, A. L.; Harris, B. J.; Neill, J. L.; Pate, B. H. An Arbitrary Waveform Generator Based Chirped Pulse Fourier Transform Spectrometer Operating from 260 to 295GHz. J. Mol. Spectrosc. 2012, 280, 3-10. https://doi.org/10.1016/j.jms.2012.07.015.

(36) Lasdon, L.; Duarte, A.; Glover, F.; Laguna, M.; Martí, R. Adaptive Memory Programming for Constrained Global Optimization. Comput. Oper. Res. 2010, 37 (8), 1500-1509. https://doi.org/10.1016/j.cor.2009.11.006.

(37) Matt Newville; Renee Otten; Andrew Nelson; Antonino Ingargiola; Till Stensitzki; Dan Allan; Austin Fox; Faustin Carter; Michał; Dima Pustakhod; Yoav Ram; Glenn; Christoph Deil; Stuermer; Alexandre Beelen; Oliver Frost; Nicholas Zobrist; Gustavo Pasquevich; Allan L. R. Hansen; Tim Spillane; Shane Caldwell; Anthony Polloreno; andrewhannum; Julius Zimmermann; Jose Borreguero; Jonathan Fraine; deep-42thought; Benjamin F. Maier; Ben Gamari; Anthony Almarza. Lmfit/Lmfit-Py 1.0.0; Zenodo, 2019. https://doi.org/10.5281/zenodo.3588521. 
(38) Park, G. B.; Steeves, A. H.; Kuyanov-Prozument, K.; Neill, J. L.; Field, R. W. Design and Evaluation of a Pulsed-Jet Chirped-Pulse Millimeter-Wave Spectrometer for the 70-102 GHz Region. J. Chem. Phys. 2011, 135 (2), 024202. https://doi.org/10.1063/1.3597774.

(39) Müller, H. S. P.; Thorwirth, S.; Roth, D. A.; Winnewisser, G. The Cologne Database for Molecular Spectroscopy, CDMS. Astron. Astrophys. 2001, 370 (3), L49-L52. https://doi.org/10.1051/00046361:20010367.

(40) Kisiel, Z.; Pszczółkowski, L.; Drouin, B. J.; Brauer, C. S.; Yu, S.; Pearson, J. C.; Medvedev, I. R.; Fortman, S.; Neese, C. Broadband Rotational Spectroscopy of Acrylonitrile: Vibrational Energies from Perturbations. J. Mol. Spectrosc. 2012, 280, 134-144. https://doi.org/10.1016/j.jms.2012.06.013.

(41) Müller, H. S. P.; Belloche, A.; Menten, K. M.; Comito, C.; Schilke, P. Rotational Spectroscopy of Isotopic Vinyl Cyanide, H2CCHCN, in the Laboratory and in Space. J. Mol. Spectrosc. 2008, 251 (1), 319-325. https://doi.org/10.1016/j.jms.2008.03.016.

(42) Mcgurk, J. C.; Schmalz, T. G.; Flygare, W. H. A Density Matrix, Bloch Equation Description of Infrared and Microwave Transient Phenomena. In Advances in Chemical Physics; John Wiley \& Sons, Ltd, 1974; pp 1-68. https://doi.org/10.1002/9780470143773.ch1.

(43) Storn, R.; Price, K. Differential Evolution - A Simple and Efficient Heuristic for Global Optimization over Continuous Spaces. J. Glob. Optim. 1997, 11 (4), 341-359. https://doi.org/10.1023/A:1008202821328.

(44) Finneran, I. A.; Holland, D. B.; Carroll, P. B.; Blake, G. A. A Direct Digital Synthesis Chirped Pulse Fourier Transform Microwave Spectrometer. Rev. Sci. Instrum. 2013, 84 (8), 083104. https://doi.org/10.1063/1.4818137.

(45) Palmer, M. Y.; Cordiner, M. A.; Nixon, C. A.; Charnley, S. B.; Teanby, N. A.; Kisiel, Z.; Irwin, P. G. J.; Mumma, M. J. ALMA Detection and Astrobiological Potential of Vinyl Cyanide on Titan. Sci. Adv. 2017, 3 (7), e1700022. https://doi.org/10.1126/sciadv.1700022.

(46) Matsuo, Y.; Minowa, T.; Komatsu, H.; Shimizu, T. Rotational- and Vibrational-state Dependence of Transverse Relaxation Rate Constants of OCS. J. Chem. Phys. 1985, 82 (7), 3127-3131. https://doi.org/10.1063/1.448209. 
(47) Patterson, D.; Doyle, J. M. Cooling Molecules in a Cell for FTMW Spectroscopy. Mol. Phys. 2012, 110 (15-16), 1757-1766. https://doi.org/10.1080/00268976.2012.679632.

(48) Green, S. Effect of Nuclear Hyperfine Structure on Microwave Spectral Pressure Broadening. J. Chem. Phys. 1988, 88 (12), 7331-7336. https://doi.org/10.1063/1.454344.

(49) Buffa, G.; Di Lieto, A.; Minguzzi, P.; Tarrini, O.; Tonelli, M. Nuclear-Quadrupole Effects in the Pressure Broadening of Molecular Lines. Phys. Rev. A 1988, 37 (10), 3790-3794. https://doi.org/10.1103/PhysRevA.37.3790.

(50) Belli, S.; Buffa, G.; Di Lieto, A.; Minguzzi, P.; Tarrini, O.; Tonelli, M. Hyperfine Level Dependence of the Pressure Broadening of $\mathrm{CH} 3$ I Rotational Transitions in the v6 = 1 Vibrational State. J. Mol. Spectrosc. 2000, 201 (2), 314-318. https://doi.org/10.1006/jmsp.2000.8106.

(51) Ball, C. D.; Mengel, M.; De Lucia, F. C.; Woon, D. E. Quantum Scattering Calculations for H2S-He between 1-600 K in Comparison with Pressure Broadening, Shift, and Time Resolved Double Resonance Experiments. J. Chem. Phys. 1999, 111 (19), 8893-8903. https://doi.org/10.1063/1.480234. 


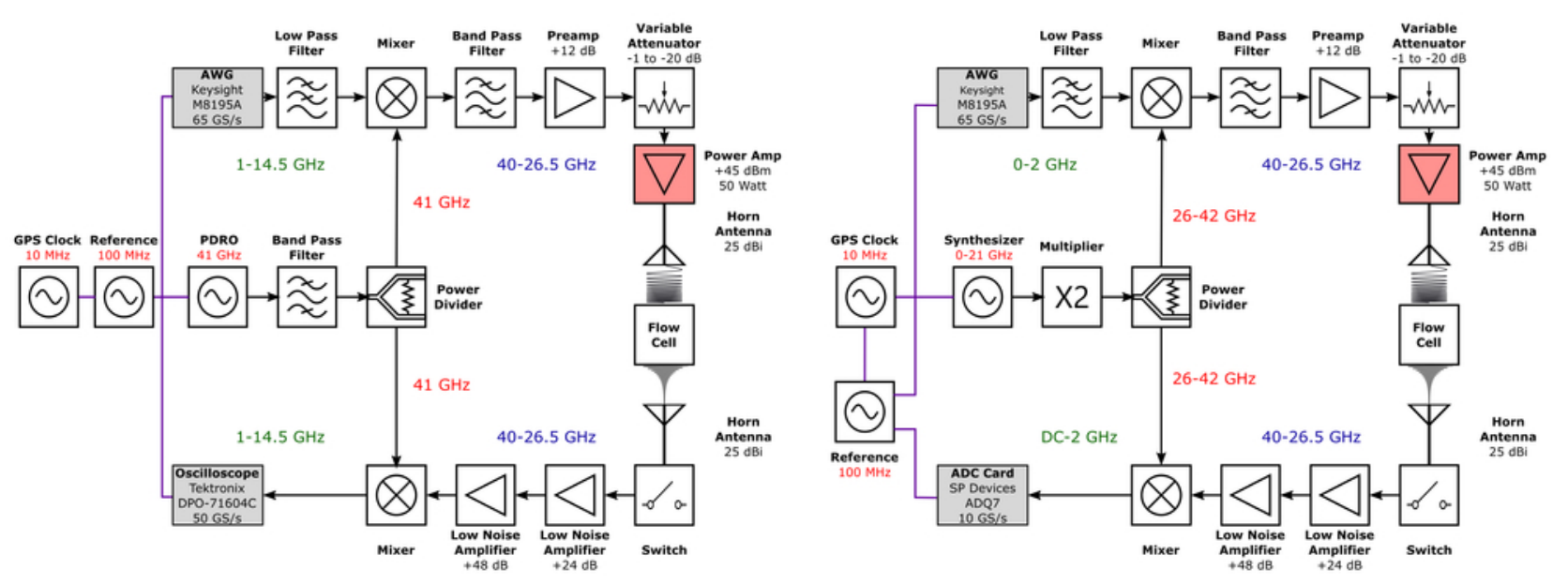

(a) - Configuration 1

(b) - Configuration 2 
(a) - Simulated Density

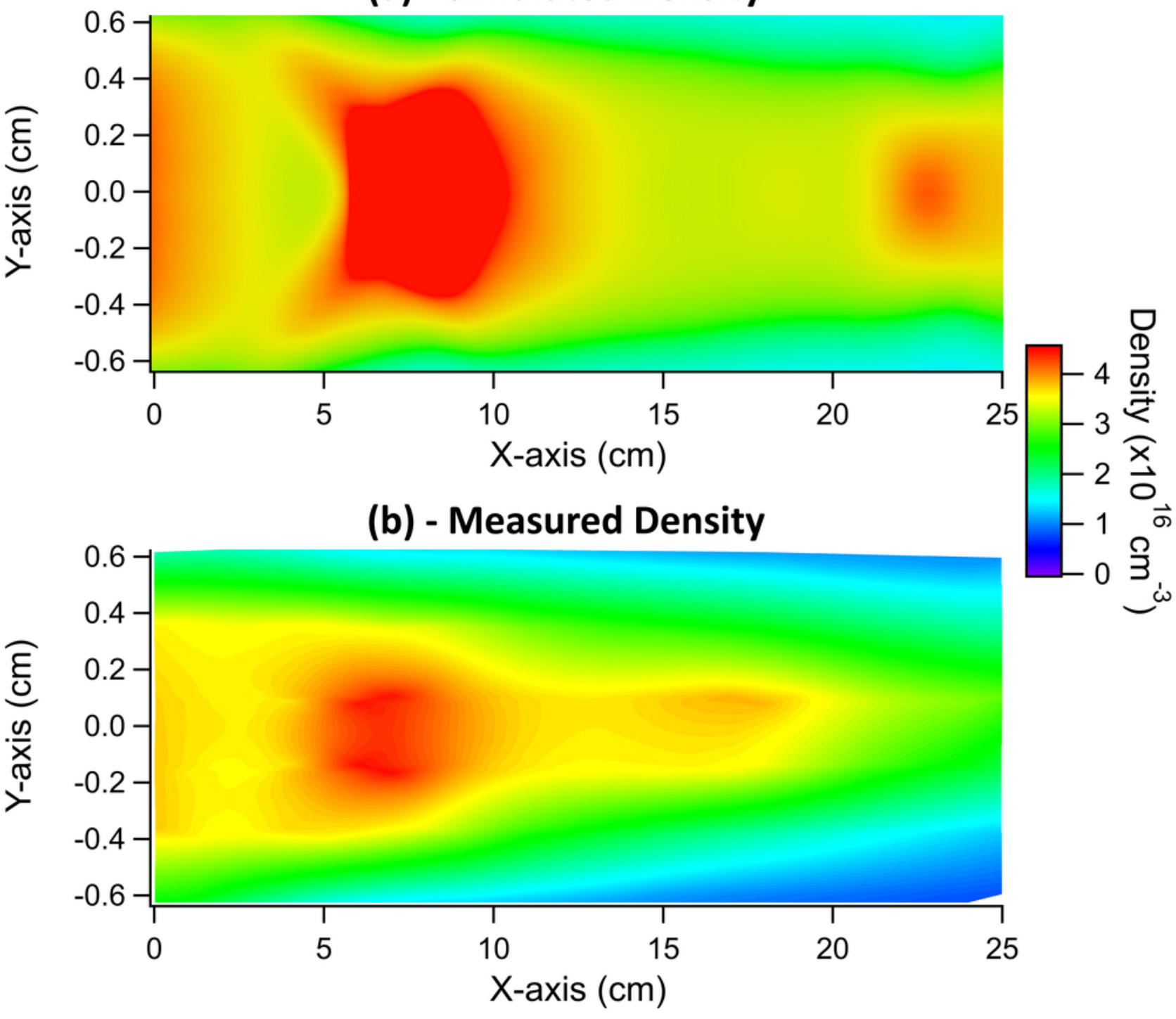

(c) - Temperature Profile

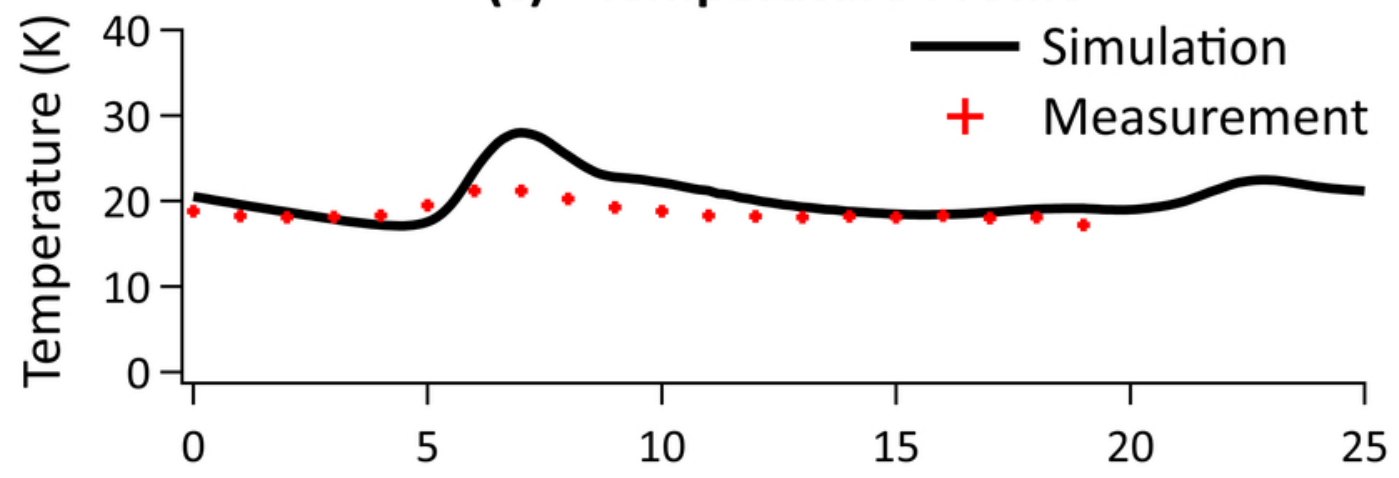

Distance from nozzle exit $(\mathrm{cm})$ 


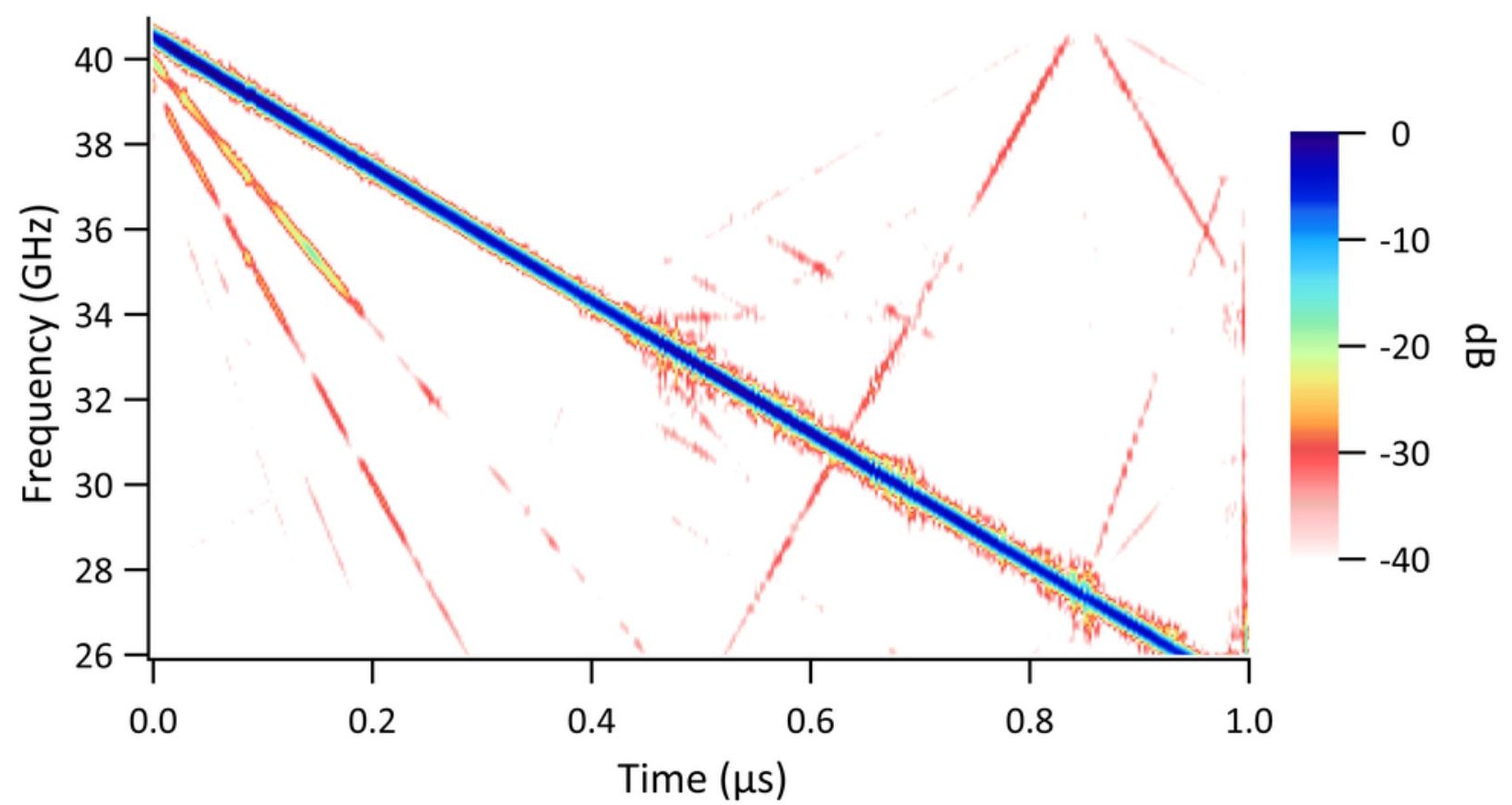




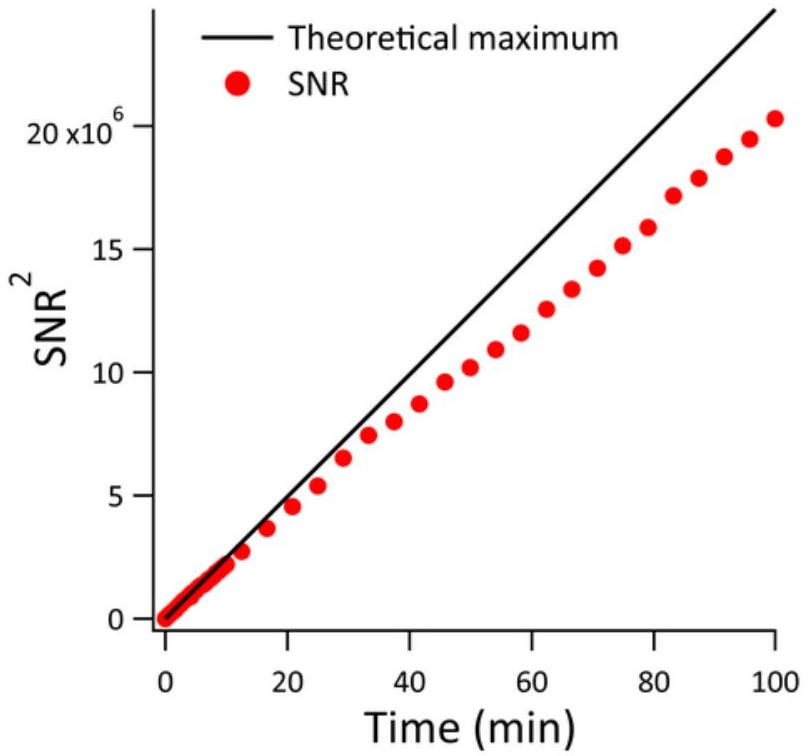




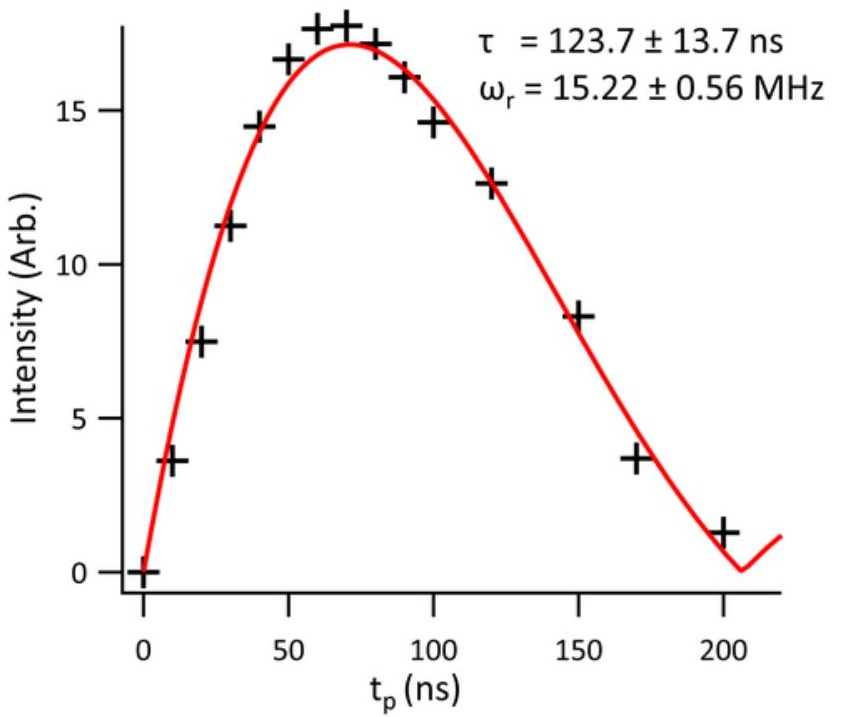




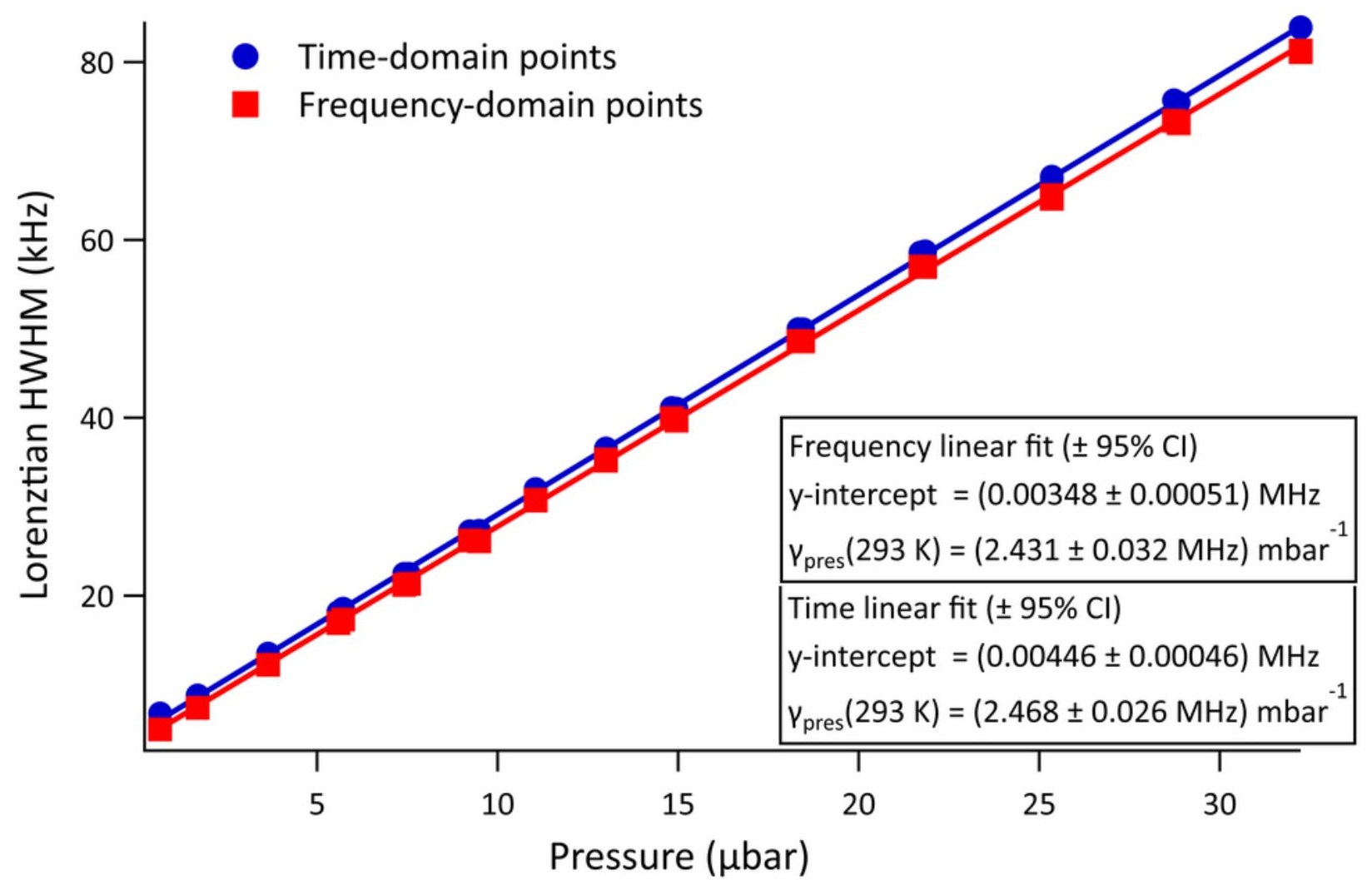


(a) - Time Domain

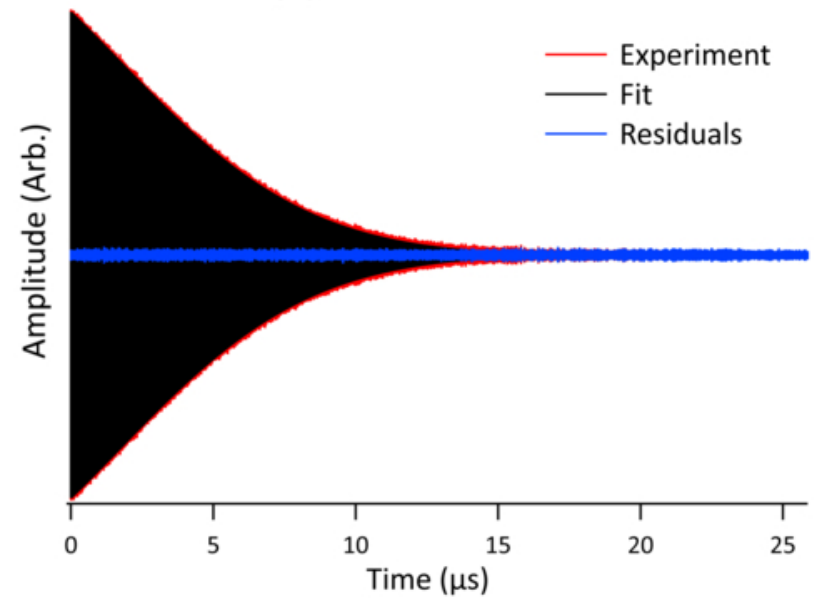

(b) - Frequency Domain
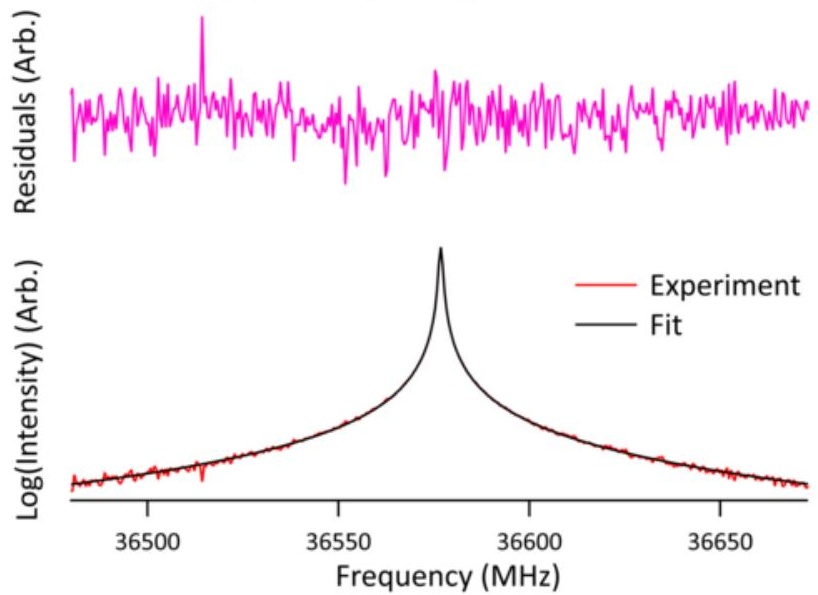


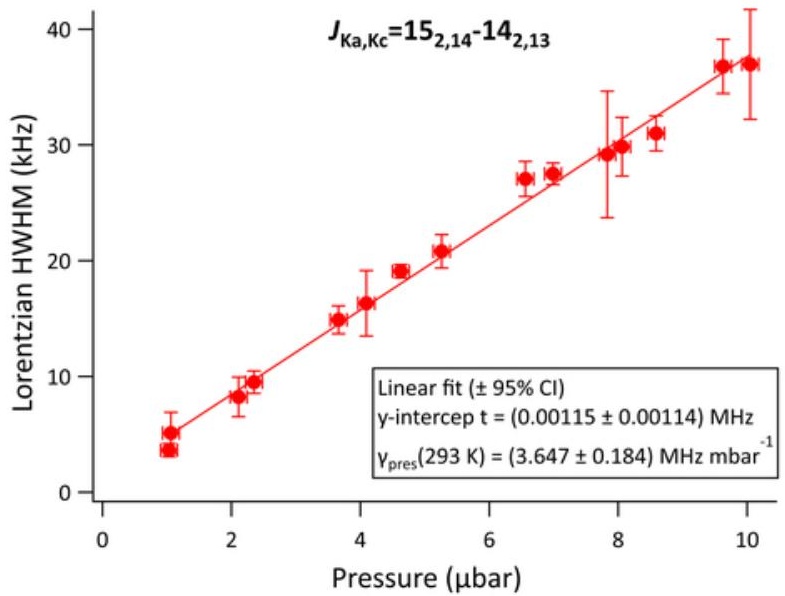




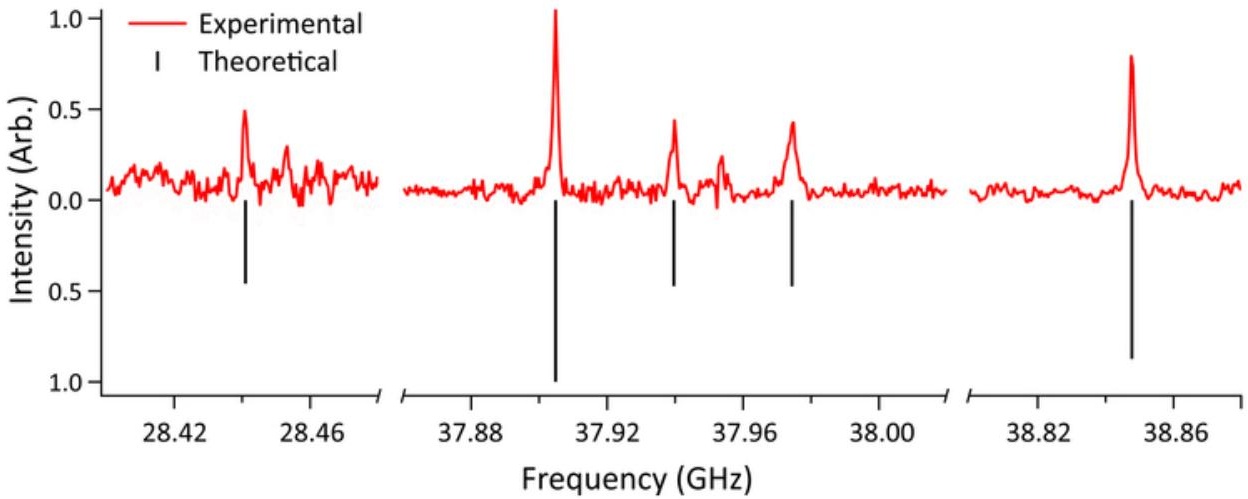




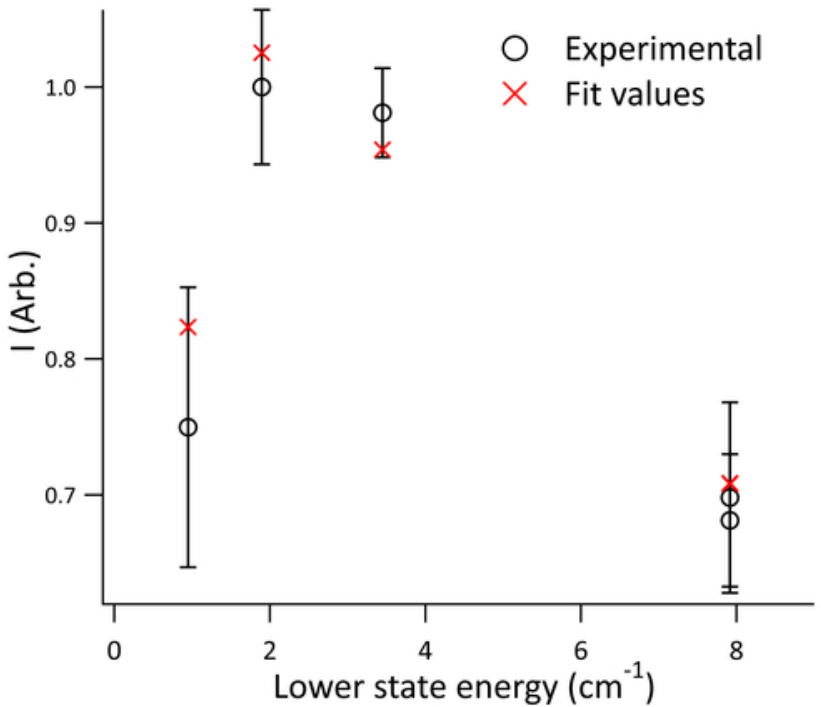


(a) - 3D representation of probed space

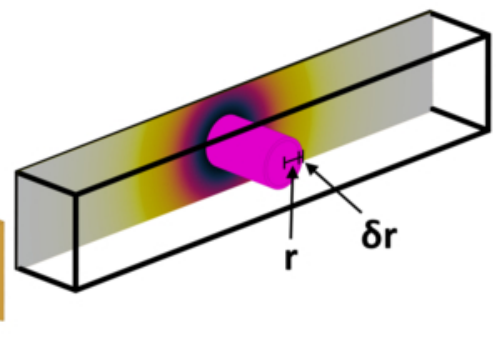

(b) - radial flow properties

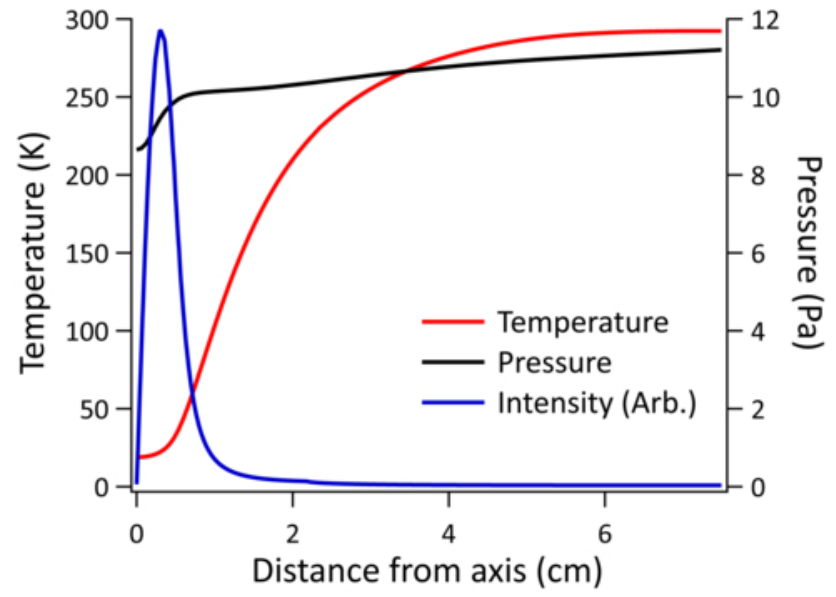

